\title{
Synchronization of great subduction megathrust earthquakes: Insights from scale model analysis
}

Matthias Rosenau ${ }^{1}$, Illia Horenko ${ }^{2}$, Fabio Corbi ${ }^{3,4}$, Michael Rudolf ${ }^{1}$, Ralf Kornhuber ${ }^{5}$, Onno Oncken ${ }^{1}$

${ }^{1}$ Department of Geomaterials, Helmholtz Centre Potsdam, GFZ German Research Centre for Geosciences, Potsdam, Germany

${ }^{2}$ Department of Mathematics and Computer Science, Università della Svizzera Italiana, Lugano, Switzerland

${ }^{3}$ Department of Science, Roma Tre University, Rome, Italy

${ }^{4}$ Géosciences Montpellier, CNRS, University of Montpellier, Montpellier, France

${ }^{5}$ Department of Mathematics and Computer Science, Free University Berlin, Berlin, Germany

Corresponding author: Matthias Rosenau (rosen@gfz-potsdam.de)

\section{Key Points:}

- We tested subduction earthquake recurrence behavior using analogue modelling

- Simulated analogue earthquake sequences mimic long-term behavior of a system of two asperities coupled by static stress transfer

- The degree of synchronization is controlled by the asperity distribution

This is the pre-peer reviewed version of the following article:

Rosenau, M., Horenko, l., Corbi, F., Rudolf, M., Kornhuber, R., \& Oncken, 0. (2019). Synchronization of great subduction megathrust earthquakes: Insights from scale model analysis. Journal of Geophysical Research: Solid Earth, 124. https://doi.org/10.1029/2018J B016597

which has been published in final form at https://doi.org/10.1029/2018J B016597. This article may be used for non-commercial purposes in accordance with Wiley Terms and Conditions for Use of Self-Archived Versions.

\section{(C) 2017.}

This manuscript version is made available under the CC-BY-NC-ND 4.0 license

http://creativecommons.org/licenses/by-nc-nd/4.0/

Please cite as:

Rosenau, M., Horenko, I., Corbi, F., Rudolf, M., Kornhuber, R. and Oncken, O. (2017): Synchronization of great subduction megathrust earthquakes: Insights from scale model analysis. SFB 1114 Preprint in EarthArXiv. pp. 135. https://dx.doi.org/10.17605/OSF.IO/PQ2U3 


\section{Abstract}

28 The size of great subduction megathrust earthquakes is controlled mainly by the number of

29 adjacent asperities failing synchronously and the resulting rupture length. Here we investigate experimentally the long-term recurrence behavior of a pair of asperities coupled by quasi-

31 static stress transfer over hundreds of seismic cycles. We statistically analyze long (c. $500 \mathrm{ka}$ )

32 time-series of M8-9 analogue earthquakes simulated using a seismotectonic scale model 33 approach with two aims: First, to constrain probabilistic measures (frequency-size 34 distribution, variability) useful for hazard assessment and, second, to relate them with 35 geometric observables (coseismic slip pattern, locking pattern). We find that the number of 36 synchronized failures (double events) relative to the number of individual failures (solo 37 events) as well as the coefficient of variation of recurrence intervals scale with the logarithm 38 of stress coupling between the asperities. Tighter packed asperities tend to recur more 39 periodically while more distant asperities show clustering. The probability of synchronized 40 failures is controlled to first order by geometrical relations (size and distance of asperities).

41 The effects of rheological properties are evident but it remains to be explored to which extent 42 they vary in nature and how sensitive the system is to those. 


\section{Introduction}

Giant magnitude 9 earthquakes unzip up to $1000 \mathrm{~km}$ long segments of active plate margins. Such long ruptures include failure of several asperities. Pre-requisites to fail synchronously (or sequentially in short succession, i.e. within seconds) are a homogeneous high stress level along the margin (i.e. in a late interseismic stage in different segments of the megathrust) and a trigger for nucelation which might be very small depending on the state of synchronization. Ruff (1996) introduced the idea of synchronization of the seismic cycle "clocks" in subduction zones by static stress transfer leading to giant earthquakes. He developed and analyzed a simple mechanical model consisting of two frictional spring-sliders coupled by a spring as an analogon of a segmented subduction zone with segments interacting by means of stress coupling (Fig. 1). He hypothesized that while individual recurrence times may initially be different (controlled by the individual frictional strength and spring stiffness) stress coupling may introduce variability and cause synchronization over multiple seismic cycles..

In a modern view Ruff's (1996) idea is based on clock advances triggered by static (Coulomb) stress transfer between asperities embedded in an elastic medium (Figure 1).

The first to model such a system realistically were Kaneko et al. (2010). They came up with a fully dynamic simulation of a pair of coseismically weakening asperities separated by a coseismically strengthening barrier. This simulation demonstrated the role of the size and rheology of the barrier in controlling rupture propagation across it. Because of the computational costs of such numerical models, the lengths of the simulated earthquakes where rather limited to few tens of cycles.

Here we realize those models by means of seismotectonic scale modelling (Rosenau et al., 2017a) which allows a realistic simulation of comparatively long analogue earthquake sequences with up to 500 individual events at a rather low experiment and time cost compared 
to numerical simulation. We simulate a subduction zone forearc wedge in an archetypical setup with two seismogenic asperities characterized by velocity-weakening and unstable stick-slip frictional behavior. The asperities are surrounded by velocity-strengthening material displaying stable creep and acting as a barrier to seismic slip. Stress coupling by means of static Coulomb stress transfer is realistically implemented by the elastic wedge and quantified using elastic dislocation modelling. While frictional and elastic properties are kept constant we vary the relative position of the two asperities along strike and across strike allowing us to explore the effects of variable stress coupling and strength contrasts between the two asperities.

Our study complements and extends recent analogue models by Corbi et al. (2017) who tested the geometric aspects of Kaneko et al. (2010) simulation using a seismotectonic scale model similar to the one we use. They were able to verify experimentally the major role of the geometric relation between the asperities in synchronization. While they were able to reproduce both the numerical results by Kaneko et al. (2010) as well as the natural observations from Japan, the significance of frictional properties remained unexplored by Corbi et al. (2017).

Here we complement these studies first by providing an analogue model with a different set of frictional properties compared to Corbi et al. (2017) to allow testing their significance more specifically. Second, we introduce a strength contrast between the two asperities, a factor which has not been tested experimentally or numerically so far. Third, we generated about 10 times longer analogue sequences (up to 0.5 Million years long including several hundreds of M8-M9 events) allowing a more rigorous statistical analysis and more reliable tests for statistical significance. All data underlying this study are published open access in Rosenau et al. (2018). 


\section{Modelling and analysis methods}

\subsection{Seismotectonic scale modelling of a subduction megathrust setting}

\subsubsection{Experimental setup and scaling}

Seismotectonic scale modelling is a cost-effective method to simulate long earthquake sequences in a fully three-dimensional, dynamic and spatiotemporally quasi-continuous framework (e.g. Rosenau et al. 2009, 2017, Corbi et al., 2013, 2017, Caniven et al. 2015, 2017). Here we recall the basics of the approach and report modifications specific to the present study.

The experimental setup used in this study is a development from an earlier quasi-twodimensional setup used for seismotectonic scale modelling by Rosenau et al. (2009, 2010) where the method has been explained in detail. The setup used in the current study is sixtimes wider and therefore truly $3 \mathrm{D}$ and allows simulating along-strike rupturing of analogue earthquakes. The experimental device consists of a glass-sided box (100 cm across strike, 60 $\mathrm{cm}$ along strike and $50 \mathrm{~cm}$ deep) with a $15^{\circ}$ dipping basal conveyer plate on top of which a compressive wedge (subduction forearc model) is set up at appropriate scale and compressed against a rigid and fixed backwall (Figure 2a).

\section{Dynamic similarity of the laboratory scale model with the natural prototype requires the ratios} of forces, which are expressed as dimensionless numbers, to be the same as in nature. We use the following set of dimensionless numbers to ensure similarity with respect to strength $\sigma$, gravity $G$, and inertia $I$ :

1. The ratio $\tau$ between gravitation and strength (either elastic, frictional or viscous) is $\tau=\rho \cdot l \cdot g / \sigma$ 
115 where $\rho$ is the rock density, $l$ is a characteristic length, $g$ is the gravitational acceleration, and

$116 \sigma$ is the elastic, frictional or viscous strength.

117 2. The Froude Number Fr relates gravitation and inertia and is

$118 \quad \mathrm{Fr}=v \cdot(g \cdot l)^{-0.5}$

119 where $v$ is a characteristic velocity.

120 3. The Cauchy Number Ca relates inertia and elasticity and is

$121 C a=\rho v^{2} / k$

122 where $k$ is the bulk modulus.

123 By keeping these dimensionless numbers the same in an experiment executed in the earth's

124 gravity field as in nature, the following scaling relationships are derived from equations (1) to

125 (3):

$126 \tau *=\tau \rightarrow(\sigma * / \sigma)=(\rho * / \rho) \cdot(l * / l)$

$127 \quad \mathrm{Fr}^{*}=\mathrm{Fr} \rightarrow\left(t^{*} / t\right)=\left(l^{*} / l\right)^{0.5}$

$128 C a^{*}=C a \rightarrow\left(k^{*} / k\right)=\left(\rho^{*} / \rho\right) \cdot\left(l^{*} / l\right)^{2} \cdot\left(t / t^{*}\right)^{2}$

129 where "*" marks the model numbers and values. The ratios between model and natural

130 prototype values are known as the scaling factors [Hubbert, 1937].

131 These scaling relationships dictate the experimental conditions and material properties (Tab.

132 1) for a given length scale and material density. The model materials used here are three times

133 less dense and designed at a length scale $\left(I^{*} / l\right)=3.3 \cdot 10^{-6}$ such that $1 \mathrm{~cm}$ in the scale model

134 corresponds to $3 \mathrm{~km}$ in nature. According to equations (4) - (6) it follows that the scale model

135 has to be weaker than the natural prototype by a factor $(\sigma * / \sigma)=1.1 \cdot 10^{-6}$ and should deform

$136 \sim 500$ times slower during analogue earthquakes in order to properly scale the body forces.

137 The corresponding coseismic time scale is $\left(t^{*} / t\right)=1.8 \cdot 10^{-3}$ (i.e. 0.1 second in the lab 
corresponds to about 50 seconds in nature). Because this dynamic time scale would result in unsuitable long recurrence intervals of analogue earthquakes in the laboratory and because

140 inertial forces can be neglected during the quasi-static inter-event time we scale the

141 interseismic periods with a factor derived from the ratio of the viscosity scale and the stress

142 scale $\left(1.3 \cdot 10^{-10} ; 1\right.$ second in the lab scales to $\sim 250$ years $)$.

143 Note that scale models represent strong simplifications of the natural prototype and their 144 application is always limited. See Rosenau et al. (2017) for a review of the seismotectonic 145 scale modelling approach.

\subsubsection{Scale model configuration and material properties}

147 The generalized subduction zone model presented here is analogous to a $300-\mathrm{km}$-wide and $148180 \mathrm{~km}$ long forearc section from the trench to the volcanic arc (Figure 2a). The scale model

149 is made up of a granular wedge of elastic-frictional plastic (elastoplastic) mixtures of EPDM 150 (ethylene propylene diene monomer) rubber pellets with refined sugar and flavored rice 151 representing the brittle forearc lithosphere. The wedge overlies silicone oil representing the 152 viscoelastic asthenosphere. We generalize the natural subduction geometry by considering a 153 planar, $15^{\circ}$-dipping megathrust between an upper plate made up of $\sim 60$-km-thick lithosphere 154 and $\sim 20-\mathrm{km}$ thick asthenosphere below the arc and an oceanic plate. The latter is represented 155 by a conveyer plate pulled constantly via a spring-loaded thrust pad at $50 \mu \mathrm{m} / \mathrm{s}$ simulating 156 plate convergence at a long-term rate of about $60 \mathrm{~mm} / \mathrm{a}$ in nature.

157 The model megathrust is defined by a few millimeters wide shear zone which forms at the 158 base of the wedge ("subduction channel", Shreve and Cloos [1986]). It is characterized by 159 rate- and state-dependent frictional behavior similar to nature [Scholz, 1998]. In particular, it 160 includes two patches $(20 \mathrm{~cm} \times 20 \mathrm{~cm} \sim 60 \mathrm{~km} \times 60 \mathrm{~km})$ displaying stick-slip deformation and 161 mimicking a pair of seismogenic asperities separated by an aseismic barrier. The friction rate162 parameter $a-b$ within the asperities, made up of rice, is $\sim-0.015$. The barrier separating the 
163 two asperities as well as up- and downdip regions of the asperities are characterized by

164 aseismic slip or stable sliding (creep) controlled by the velocity strengthening behavior ( $a-b \sim$

$165+0.015)$ of frictional slip in sugar. Material properties of this seismotectonic scale model have

166 been documented in detail in Rosenau et al. [2009, 2017] and Rudolf et al. (2016) and are

167 reported in Table 1.

168 The two asperities have an along subduction zone strike center-to-center distance (hereafter

169 called spacing) $\mathrm{dx}$ and are a relative shift across subduction zone strike (hereafter called

170 offset) dy (Figure 2b). This configuration allows exploring the effects of stress coupling (as

171 defined below in section 2.2.2) as well as strength contrast. We define the latter as the shear

172 strength of the weaker (shallower) asperity 2 relative to the stronger (deeper) asperity 1 :

173 Strength contrast $=$ Tau2/Tau 1

174 Strength contrast therefore ranges theoretically from close to 0 to 1 . Note the somewhat 175 counter-intuitive effect that low strength contrasts are reflected by Tau2/Tau1 values. In total

17612 configurations have been realized in which we vary the strength contrast from 0.6 to 1.0

177 and the stress coupling from a few ppm to percent (Fig. 2c). The experimental runs took place

178 under normal gravity conditions and in a dry room climate $\left(22-23^{\circ} \mathrm{C}, 30-40 \%\right.$ humidity $)$.

\section{$179 \quad$ 2.1.3 Experimental Monitoring and Strain Analysis}

180 For strain analysis of the evolving model wedges we use an optical image acquisition and

181 correlation system (particle image velocimetry, PIV StrainMaster by LaVision, Germany, see

182 Adam et al. [2005], Rosenau et al. [2009, 2010, 2017] for applications in analogue tectonic

183 and earthquake simulation).

184 During an experiment, the locations of particles on the model surface (i.e. within the $x-y$-plane

185 of the model, Fig. 2) are recorded by sequential 11 Mpx-digital images of a 14-bit 186 monochrome charge-coupled device (CCD) camera acquired at a frequency of $10 \mathrm{~Hz}$. The $x^{-}$ 
$187 y$-displacement vector field between successive images is then determined by cross188 correlation of textural differences (i.e. gray values) formed by groups of particles using a Fast 189 Fourier Transform algorithm. The spatial resolution of the final displacement vector grid is

$1903 \mathrm{~mm}$ or about $1 \mathrm{~km}$ in nature. For each grid-cell, an average $x$-z-displacement vector is

191 determined at micrometer precision ( decimeter scale in nature). This allows for observing

192 episodic surface deformation events corresponding to earthquakes of moment magnitude $M_{w}$

$193>8$. Analogue earthquakes are characterized by episodic, usually more than one order-of-

194 magnitude increased strain rates and a change in polarity of the wedge deformation from

195 "landward" motion (in negative y-direction) and compaction during the interseismic stage to 196 "seaward" motion and extension during the coseismic stage (Figure 3 a, b). Earthquakes 197 typically occur within a 0.1-second time interval, i.e. are captured by a solo image.

\section{$198 \quad 2.2$ Elastic dislocation modelling}

199 We use elastic dislocation modelling following Okada (1992) and Okada (1985) for 200 coseismic slip inversion and Coulomb stress transfer calculation employing the Matlab-based 201 software package "Coulomb" by Toda et al. (2011, Coulomb 3.3 Graphic-rich deformation 202 and stress-change software for earthquake, tectonic, and volcano research and teaching - user 203 guide: U.S. Geological Survey Open-File Report 2011-1060, 63 p., available at 204 http://pubs.usgs.gov/of/2011/1060). The model setup for elastic modelling uses the scaled 205 values of geometric and mechanical parameters given by the analogue model.

\subsubsection{Slip inversion}

207 Surface deformation during analogue earthquakes as captured by PIV is converted into 208 coseismic slip along the megathrust using inversion factors derived by forward elastic 209 dislocation modelling. Accordingly we find the factors relating horizontal surface deformation 210 UY directly above the dislocation at depth to slip S along it to range between 0.2 and 0.5 
211 depending non-linearly on the depth of dislocation (Figure A1). Shallow dislocations show

212 larger factors, i.e. are less attenuated. We do not aim at a formal inversion or distributed slip

213 modelling. Instead we consider here mean coseismic surface displacement over the projected

214 surface area of the asperity to be a valuable proxy for mean coseismic slip over the asperity at 215 depth.

\section{$216 \quad$ 2.2.2 Stress coupling}

217 For quantifying the interaction by means of stress coupling between the asperities we follow 218 the principles of static Coulomb stress transfer (CFS) modelling as established by King et al. 219 (1994) Toda and Stein (2002) and Lin and Stein (2004).

The model setup for CFS modelling is such that we impose thrust slip on one asperity (trigger asperity) and average the predicted CFS increase (dCFS) for thrust faulting on the receiver asperity (Fig. 2a). We then define a parameter called stress coupling as the CFS increase averaged over the receiver asperity normalized by the stress drop on the trigger asperity:

224 Stress coupling $=\mathrm{dCFS} / \mathrm{dTau}$.

225 In the present setup stress coupling is in the order of less than a ppm up to one percent similar 226 to nature. Stress coupling falls off exponentially with distance and varies non-linearly across227 strike of the megathrust as a function of asperity spacing (dx) and offset (dy, Fig. A2).

\section{$228 \quad 2.3$ Numerical analysis of surface deformation time series}

229 Experimental time-series of surface deformation consist of typically a sequence of 30.000 230 images and corresponding incremental vector fields. To detect analogue earthquakes from 231 such a big data set we usually rely on computational algorithms sensitive to accelerations 232 validated by visual inspection. However, because of experimental noise such a kinematic 233 approach based on thresholding velocity usually has a high detection limit. Instead of 
234 thresholding velocities to detect earthquakes stages we here employ a numerical time-series 235 analysis technique developed in computational statistics. This allows us to detect events 236 which can be below the detection threshold of classical kinematic approaches.

237 As input we use the surface deformation time-series of mean across-strike velocities UY_1(t) 238 and UY_2(t) in the surface projection area of the two asperities (Figure 3c). Those data 239 typically show a transient phase without much activity in the beginning which reflects stress 240 buildup and reorganization within the analogue model (Figure 3c). After about 5.000-10.000 241 time-step increments (500-1000 seconds) surface accelerations reflecting analogue 242 earthquakes start to occur with increasing size and frequency and quickly reach a quasi243 stationary state. We use observations from this quasi-stationary state for further analysis.

244 To analyze the obtained experimental time series, we deploy a nonparametric time series 245 analysis methodology called Finite-Element-Method with Bounded Variation of model 246 parameters (FEM-BV) (Horenko 2009, Horenko 2010, Metzner et. al. 2012). Although it is 247 computationally more expensive then the common methods, FEM-BV has several important 248 conceptual advantages that were recently illustrated for various time series analysis 249 applications in geosciences (Vercauteren et. al. 2015, Risbey et. al 2015, Franzke et. al. 2015, 250 Kaiser et. al. 2015, O'Kane et. al. 2016). This nonparametric method is automatized, does not 251 rely on any tunable user-defined parameters (like thresholds values for the event 252 identification) and allows to go beyond strong parametric assumptions (like linearity, Gauss 253 or Poisson distribution assumptions for observed densities, stationarity or Markovianity) 254 assumptions that are a constitutive part of the more common statistical time series analysis 255 approaches like multilinear regression, Hidden Markov Models or clustering methods (e.g. 256 Shearer and Stark, 2012). Going beyond these assumptions is especially important since 257 analyzed data exhibits a strong regime-transition behavior, is non-stationary, non-Markovian and non-Gaussian in the regimes. Moreover, defining ad hoc threshold values for the events 
259

260

$\mathrm{CV}=$ standard deviation / mean.

275 CV serves as a first-order proxy for recurrence behavior: a CV of 1 characterizes a random 276 behavior while $\mathrm{CV}<1$ suggests characteristic or periodic recurrence. $\mathrm{A} \mathrm{CV}>1$ is characteristic 277 of clustering (e.g. Kuehn et al., 2008, Rosenau and Oncken, 2009).

could potentially introduce a user-defined bias. We refer to Metzner et. al (2012) for mathematical/statistical details of the FEM-BV methodology - as well as for its computational comparison with more common time series analysis methodologies.

\subsection{Statistical analysis of analogue earthquake sequences}

Based on the long sequences of analogue earthquakes we explore the recurrence behavior and its intrinsic variability by means of univariate and bivariate statistics.

A simple measure of probability, used by earlier studies as well, is the relative number of events of a given character (e.g. solo events, double/synchronized events). To get further insight into the statistics however, the present studies allows producing probability distribution functions (pdf) of distinct event parameters. We here use the pdf of moment magnitudes (Figure 4a and A3) to characterize the "Gutenberg-Richter" frequency-size relationship. And we use the pdf of the recurrence interval time (Figure $4 \mathrm{c}$ and A3) to differentiate between periodic and aperiodic (e.g. clustered) occurrence of events.

Moreover, we quantify variability of the seismic moment (M0) and recurrence time (Trec) by calculating the associated coefficients of variation:

\footnotetext{
fof
}

\section{Experimental observations and interpretations}

\subsection{Seismic performance of the scale model}

A typical earthquake catalogue simulated by our scale model consist of up to 500 events of moment magnitude 8-9 which occur over a time-period of about 500 ka (Fig. 4a). M8 events 
282

usually involve only one asperity while a synchronous failure of both asperities usually results 283 in the M9 events. Analogue earthquakes are always followed by afterslip lasting for not more 284 than one frame (0.1 s) surrounding the asperities (Figure $3 \mathrm{a}, \mathrm{b}$ ). Generally the shallow 285 asperity generates more surface displacement than the deep one: This is related to static effects as predicted by elastic dislocation modelling (Figure A1). The picture inverts when the correction for depth of dislocation is applied. Then, deeper asperities show larger slip. This is consistent with higher loads causing higher frictional strength at greater depth as predicted by Mohr-Coulomb theory. As a consequence, the deeper asperities are mechanically stronger and able to accumulate more slip deficit in the interseismic period compared to the shallow asperities.

We refer to slip events which occur on both asperities within one time frame $(0.1 \mathrm{~s})$ as double or synchronized events. If the second event occurs independently within the next frame, we refer to it as an aftershock or a clustered event. A minority of aftershocks are actually relatively small normal faulting events. We interpret those as a result of dynamic overshoot during the preceding thrust event. Normal events occur almost exclusively in the shallow asperity. We include those rare normal events in our analysis since they represent an integral part of the long-term slip budget. Accordingly, they show up with a negative seismic moment in Figure 4a.

When analyzing synchronous (double) events, clustered (solo) events and normal events (overshoots) as a function of stress coupling dCFS/dTau and strength contrast Tau2/Tau1 a clear picture emerges (Figure 5). Accordingly, a synchronous double events increase in number from 20 to $80 \%$ as stress coupling increases by two orders of magnitude (from less than a ppm up to a percent). At the same time, clustered events decrease. This simply reflects a higher degree of synchronization in strongly coupled systems. Overshoots show no clear correlation with stress coupling but a negative correlation with strength contrast (Figure 5). 
307 This is consistent with overshoots occurring preferentially in shallow regions of the wedge.

308 Both synchronous double and clustered solo events show no correlation with strength

309 contrast. An apparent increase of the range of proportion of those events with stress contrast

310 reflects the systematically wider range in stress coupling realized for lower strength contrasts.

\section{$311 \quad 3.2$ Frequency-size distributions}

312 Frequency-size distributions of simulated earthquakes share similar shapes. The pdfs of 313 moment magnitude are generally skewed negatively (towards the left) and very peaked as 314 exemplified in Figure 4b. The PDFs of recurrence times are generally bimodal characterized 315 by a peak at short periods ( $0.1 \mathrm{sec}$ or 25 years) and a quasi-normally distributed bump around 316 the mean recurrence time as exemplified in figure $4 \mathrm{c}$.

317 Plotting mean recurrence times and mean seismic moments and their variability in terms of $318 \mathrm{CV}$ into the parameter space (Figure 6) shows the following: Mean recurrence time and 319 seismic moment both increase with an increase in stress coupling. At the same time their CVs 320 decrease. $\mathrm{R}^{2}$-values for these correlations range between 0.3 and 0.6 (Table A1 in appendix) 321 and the trends considered significant.

322 We interpret this correlation of $\mathrm{M}_{0}$ and $\mathrm{T}_{\text {rec }}$ with stress coupling as reflecting a dynamic 323 interaction causing higher slip in case of more strongly coupled asperities. Larger slip 324 consistently lengthens the interseismic period resulting in longer recurrence times. The 325 increase in size seems also to have a positive effect on the periodicity with larger stress drops 326 regulating the earthquake cycle thus decreasing the $\mathrm{CV}$ to 0.5 .

327 A weak positive correlation exist between $T_{\text {rec }}$ and strength contrast $\left(R^{2}=0.25\right)$. Accordingly, 328 earthquake frequency increases as the weak asperity becomes weaker. We interpret this as 329 being a behavior predicted by Ruff (1996) where the weaker asperity, which has intrinsically 
330 the shorter recurrence time, causes clock advance of the stronger asperity, which has

331 intrinsically longer recurrence times. A correlation between $\mathrm{M}_{0}$ as well as the associated CVs

332 with strength contrast have not been observed to be statistically significant $\left(\mathrm{R}^{2}<0.05\right)$.

333 The significant trends of $\mathrm{M}_{0}$ and $\mathrm{T}_{\text {rec }}$ with dCFS/dTau are replotted in Figure 7 with a 334 differentiation between all events (solo and double events) and solo events to explore the 335 effects of stress coupling on the frequency-size distributions in more detail. Consistently, 336 considering only double events increases mean seismic moment and mean recurrence time 337 and decreases the associated CVs. This is simply a result of setting a magnitude threshold.

338 More interestingly, however, is the observation that the trends differ for the two groups of 339 events: For example, the positive correlation of Trec with stress coupling observed for all 340 events is inverted to a negative correlation if only double events are considered (Fig. 7a). This 341 is simply the result of double events being systematically rarer in more weakly coupled 342 systems as has been predicted by Ruff (1996). At the same time, recurrence times of double 343 events are more sensitive to stress coupling than the recurrence times of all events: Double 344 events recur almost randomly for weakly coupled systems and periodically for strongly 345 coupled systems as suggested by a $\mathrm{CV}$ of $T_{\text {rec }}$ ranging between 1 and 0.1 . On the other side, 346 the $\mathrm{CV}$ of $M_{0}$ is much smaller (0.2) and independent of stress coupling indicating a 347 characteristic size of double events.

\section{Discussion}

349 4.1 Relation between asperity distribution and recurrence behavior: A characteristic $350 \quad$ length scale in nature?

351 Based on experimentally simulated long subduction earthquake records we are able to 352 constrain the intrinsic variability of subduction earthquakes in terms of size and recurrence 
353 times and shed light on their relationship to the distribution of asperities. Rosenau et al.

354 (2017) showed that the transition from one to two asperities involves a principle change from 355 periodic (Recurrence time's $\mathrm{CV}=0.2)$ towards more randomly occurring earthquakes $(\mathrm{CV}=$ 356 1). This is consistent with spring-slider models suggesting a single isolated spring-slider 357 system to be periodic while a coupled pair of spring-sliders shows a more complex behavior 358 (e.g. Ruff, 1996). The system simulated here shows a strong correlation between the coupling 359 (controlled by asperity distribution) and recurrence variability increasing from 0.2 to 1 as 360 coupling decreases (Fig. 7 c). This range spans a considerable larger range than what is seen 361 in natural examples which is usually characterized by a $\mathrm{CV}<0.4$ :

362 For example, the Holocene tsunami record offshore western North America suggest that great 363 M9 Cascadia subduction zone earthquakes have occurred about every 500 to 600 years during 364 the past $10 \mathrm{kyr}$ (Goldfinger etal., 2003) with a CV of 0.36-0.39 (Sykes and Menke, 2006). For 365 the Nankai trough, Sykes and Menke (2006) report a CV of 0.26-027. In the Northern Chile366 Southern Peru seismic gap which last broke in 1877 (M8.8) the reported historical recurrence 367 interval for the past 500 years has been estimated at $111+/-33$ years (Comte and Pardo, 368 1991) resulting in a CV of 0.3. Similarly, in southern Chile, in the area of the great 1960 and 3692010 earthquakes, leveling and dating of Holocene strandlines by Bookhagen et al. (2006) 370 suggests that great earthquakes have occurred every $180 \pm 65$ years over the last 3 to $4 \mathrm{kyr}$, 371 from which a $\mathrm{CV}=0.36$ can be calculated.

372 Although the data base is limited, this rather narrow range of low CVs in nature in 373 combination with the here suggested causal link between $\mathrm{CV}$ and asperity distance let us 374 speculate that there might be a characteristic length scale in the asperity distribution in nature. 375 In our models a $\mathrm{CV}<0.4$ is reached only by the narrow configurations where barriers between 376 asperities are significantly smaller than the asperities themselves. Such a narrow asperity 377 configuration can be found for example in the region of the 1960 and 2010 Chile earthquakes 
378 (Moreno et al., 2009, 2010, 2011). More examples can be found e.g. in Hayes (2019) finite

379 fault model data base, however, a rigorous review of natural examples with respect to this 380 aspect is beyond the scope of this paper.

\subsection{Predicting asperity interaction: Towards proxies for barrier efficiency}

382 We simulated long time-series of analog subduction megathrust earthquakes in order to 383 constrain the recurrence pattern of a simple system with two asperities coupled by static stress 384 transfer. Similar experiments (Corbi et al. 2017) and numerical simulations (Kaneko et al 385 2010) have been carried out to find the critical parameters controlling the probability of a 386 rupture bridging the barrier and causing a synchronized failure of the asperities. We here add experimental data representing a different set of material parameters and geometries which allows testing the existing concepts and to identify the minimum set of parameters needed.

Kaneko et al. (2010) suggested a set of parameters combined in a proxy for barrier efficiency called $\mathrm{B} . \mathrm{B}$ is the ratio of the stress increase required to bridge the barrier to the coseismic stress drop. B included parameters which are directly and indirectly (involving assumptions) observable in nature (geometric, kinematic, dynamic and friction parameters). Given the complexity of B and the uncertainty in the choice of some of the parameters included (e.g.

394 frictional parameters), Corbi et al. (2017) aimed at a more simple proxy based solely on first395 order geometric relationships easy to observe in nature, i.e. the barrier-to-asperity length ratio $396 \mathrm{Db} / \mathrm{Da}$. With respect to these two proxies, we consider the stress coupling as defined here as a 397 proxy for barrier efficiency of intermediate complexity. Similar to $\mathrm{Db} / \mathrm{Da}$, stress coupling can 398 be inferred primarily from geometric observations (size and location of asperities).

399 In Figure 8 we compare the three proxies based on the setup presented in this study. 400 Obviously, there is a good correlation between stress coupling, B and $\mathrm{Db} / \mathrm{Da}$. $\mathrm{Db} / \mathrm{Da}$ seems 401 slightly more sensitive to stress coupling than B as suggested by its steeper slope. In any case, 
402 a correlation coefficient $\left(\mathrm{R}^{2}\right)$ of 0.6 to 0.8 suggests general interoperability of the three 403 proxies.

404 Figure 9 shows the collapse of all existing experimental, numerical and real world data in a 405 plot of percentage synchronized ruptures (double events) versus B while plotting those data 406 against $\mathrm{Db} / \mathrm{Da}$ separates the data into roughly parallel trends. Because the data used represent 407 a wide spectrum of geometrical and rheological parameters, the collapse indicates the 408 versatile nature of the proxy B for anticipating double events.

409 On the other hand, the systematic offset trends suggest that while $\mathrm{Db} / \mathrm{Da}$ seems to allow for a 410 strong control on synchronization, material properties cannot be neglected. For instance, it 411 appears that the setup used in the present study generates double events more easily. While 412 for the experiments by Corbi et al. (2017) and the natural example a threshold for double 413 events at $\mathrm{Db} / \mathrm{Da}$ of 0.5 emerges, in the experiments presented here this threshold is 414 significantly higher ( $>1)$. This suggests that the barrier in the Corbi et al. (2017) experiments 415 as well as in the Nankai area are mechanically more effective than in our setup.

416 We conclude that for the moment, the full complexity of the proxy B by Kaneko et al. (2010)

417 is needed to account for the variability of mechanical parameters present in the experiments. 418 To which extent these parameters vary in nature and therefore control the threshold value of $419 \mathrm{Db} / \mathrm{Da}$ remains to be explored.

\section{$420 \quad 5$ Conclusions}

421 Based on experiments generating long time-series of analog subduction megathrust 422 earthquakes we explored the process of interaction and synchronization of two velocity423 weakening asperities separated by a velocity-strengthening barrier. We found the following: 
- Synchronization is controlled by the static stress transfer from a one asperity to the other, quantified by the stress coupling dCFS/sTau. Accordingly, the percentage of synchronized events scales with the logarithm of (normalized) Coulomb stress change on the receiver asperity.

- A strength contrast between the two asperities has no significant effect on synchronization but decreases the recurrence time of double events because the weaker asperity dictates the recurrence intervals.

- Analogue earthquakes in strongly coupled systems (narrower asperity distribution) recur more periodically and with a more characteristic size than in weakly coupled systems.

- A narrow asperity distribution might be typical for natural subduction zones characterized by quasi-periodic recurrence

Three proxies for the barrier efficiency, B (Kaneko et al. 2010), Db/Da (Corbi et al., 2017) and the newly defined stress coupling have been cross-validated and tested for applicability:

- $\mathrm{Db} / \mathrm{Da}$ is the most simple and easiest to apply proxy and incorporates the most sensitive parameters to work first-order. It relies on geometries which - if they are stationary over multiple seismic cycles - we are able to constrain using interseismic locking and paleoseismological observations.

- $\mathrm{B}$ is the most versatile proxy and it captures the physics - but several parameters are not well constrained or uncertain in nature.

- Stress coupling is of intermediate complexity and interoperable with $\mathrm{Db} / \mathrm{Da}$ and $\mathrm{B}$.

In order to arrive at a minimum set of parameters necessary to describe seismic hazard in subduction zones we suggest to further explore the variability of those parameters in B 
448 which are not well known in nature, to define the sensitivity of simpler proxies and to aim 449 at constraining their upper and lower bounds.

450 


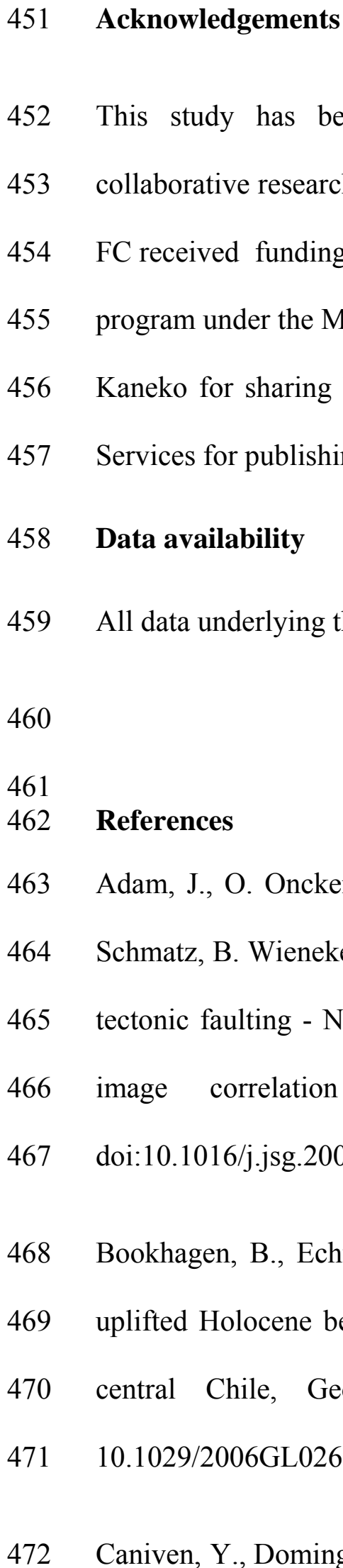

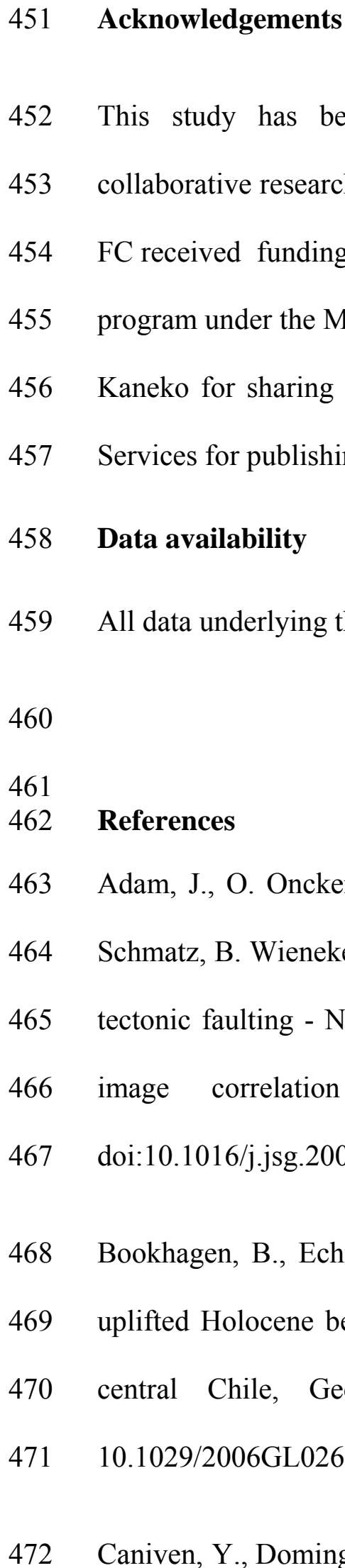

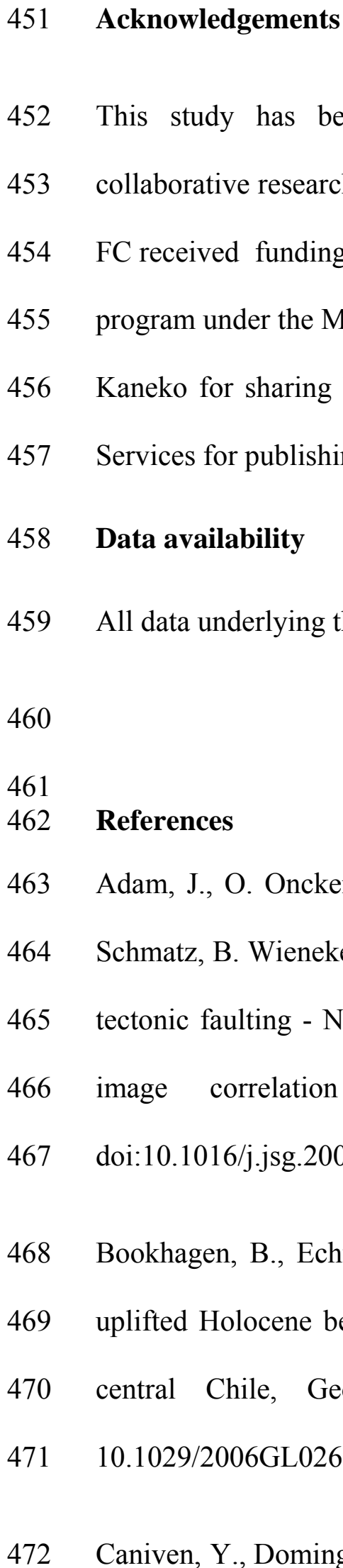

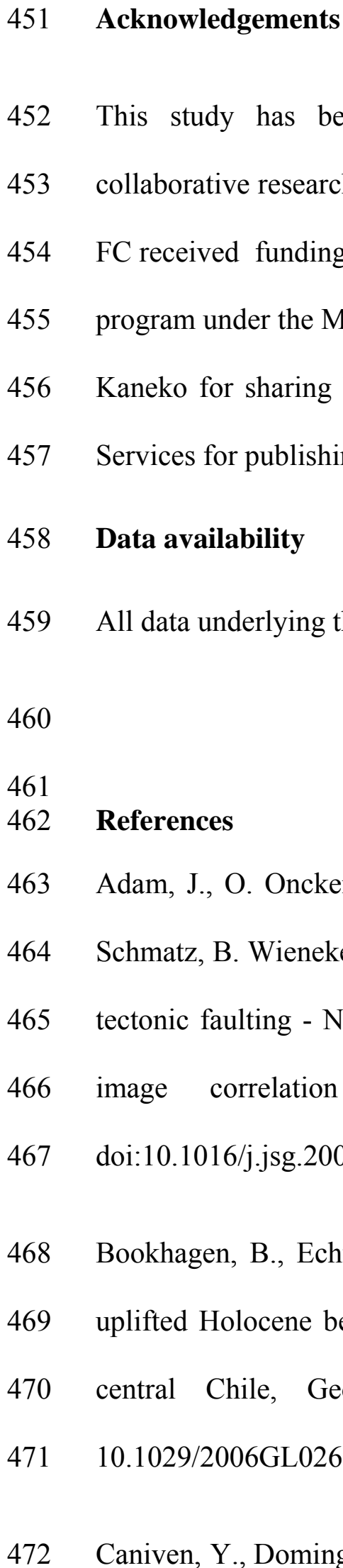

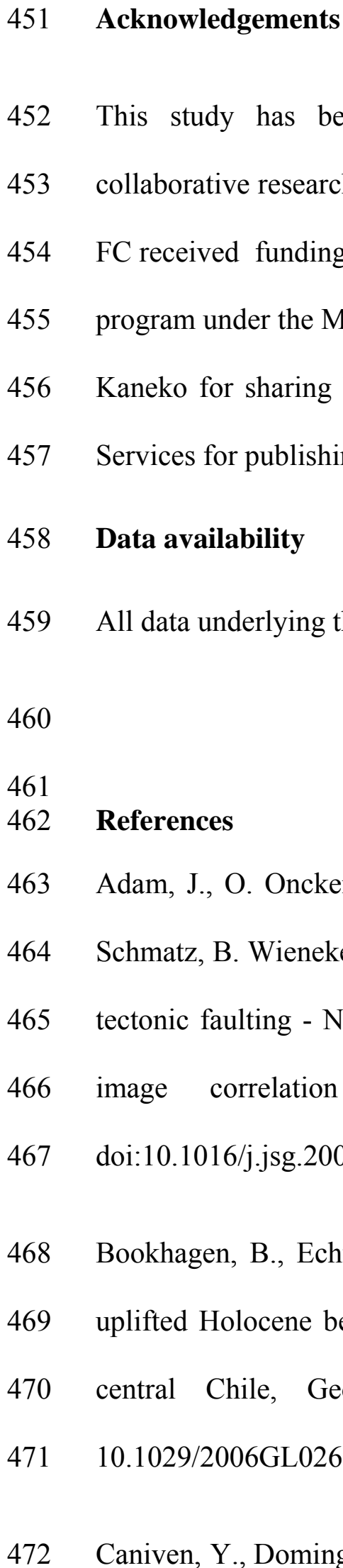

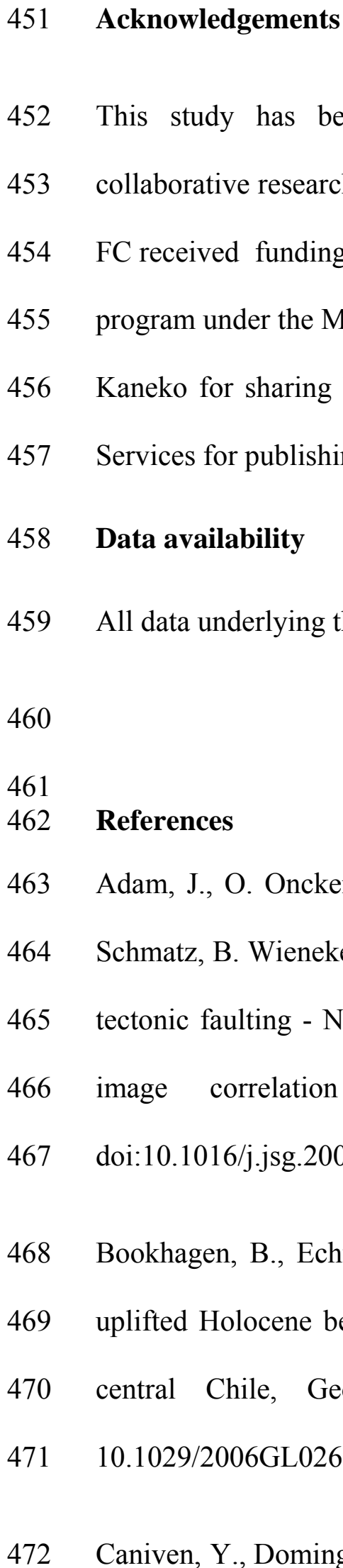

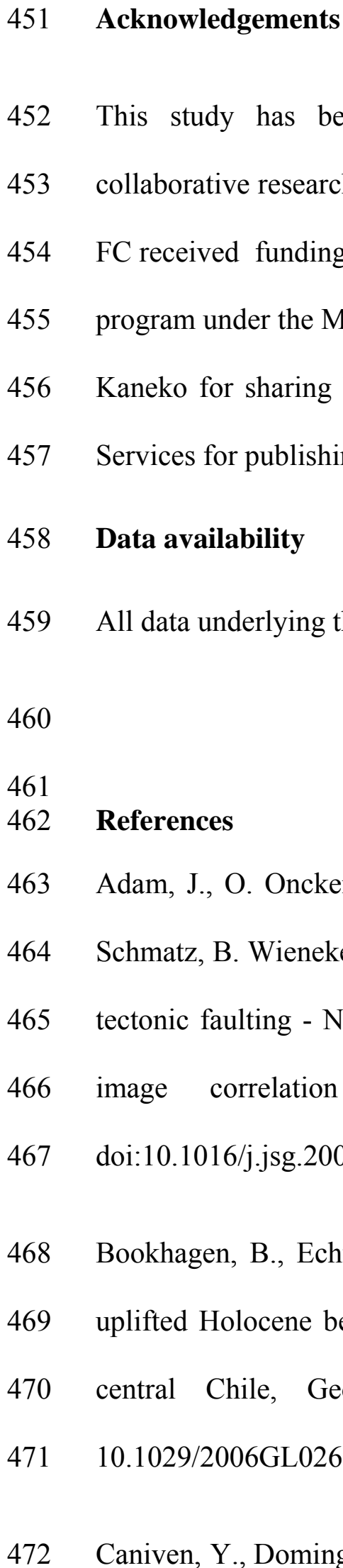

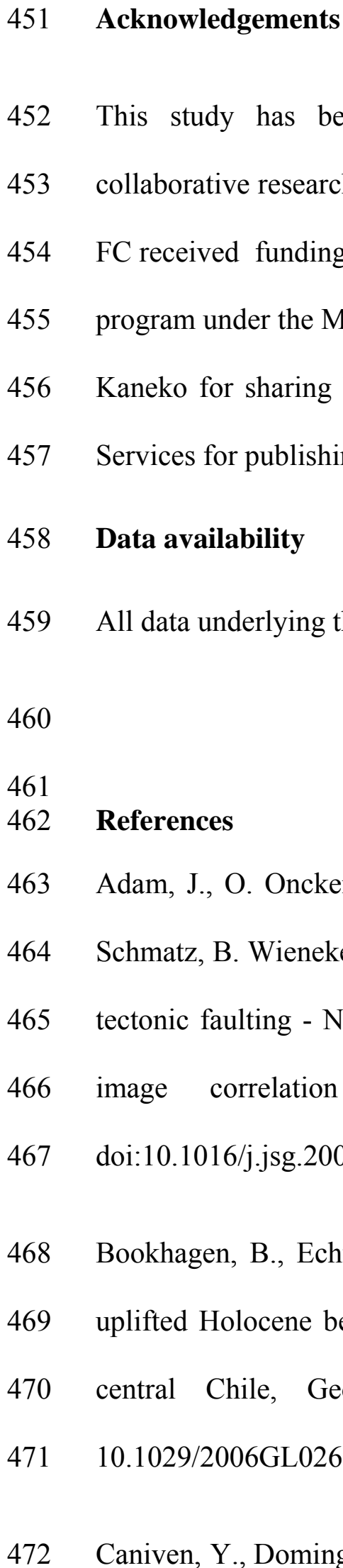

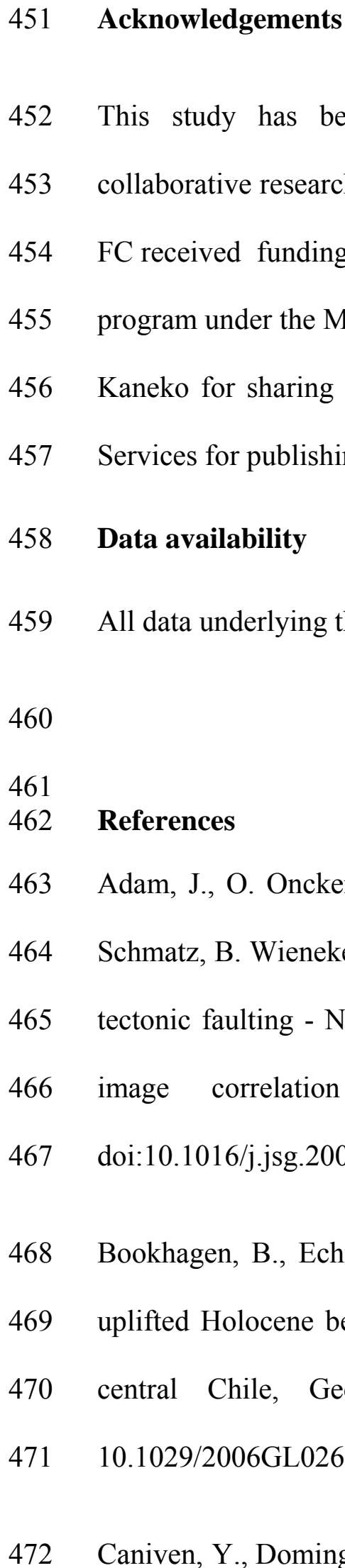

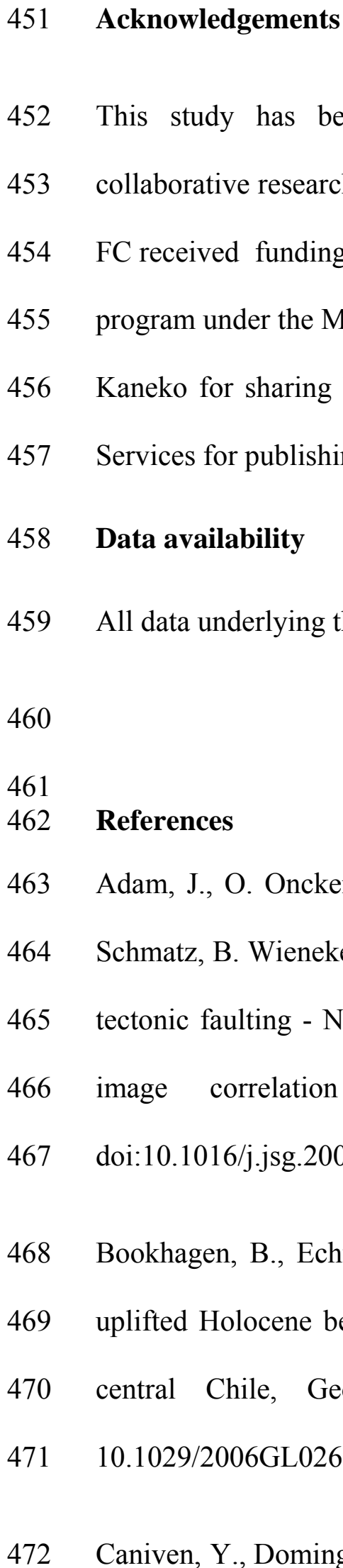

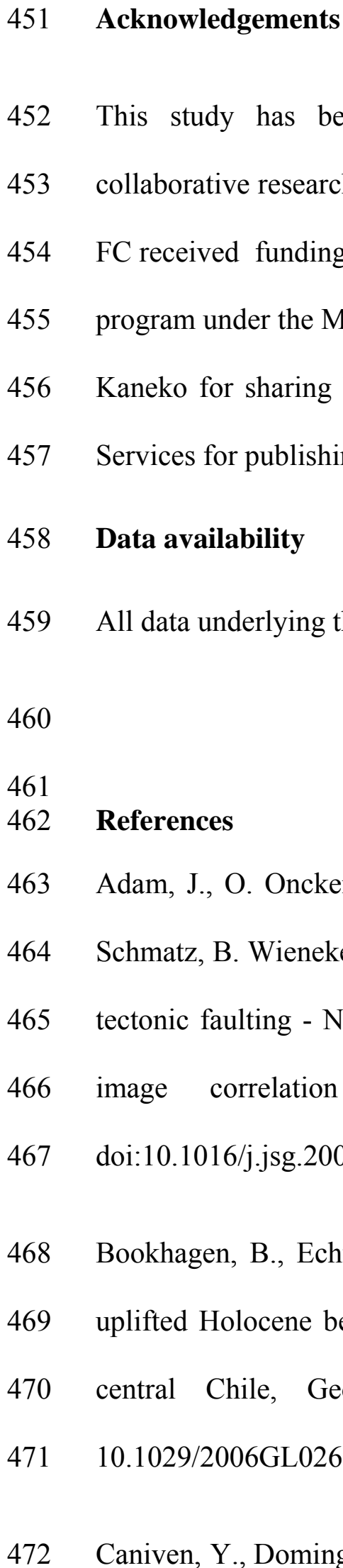

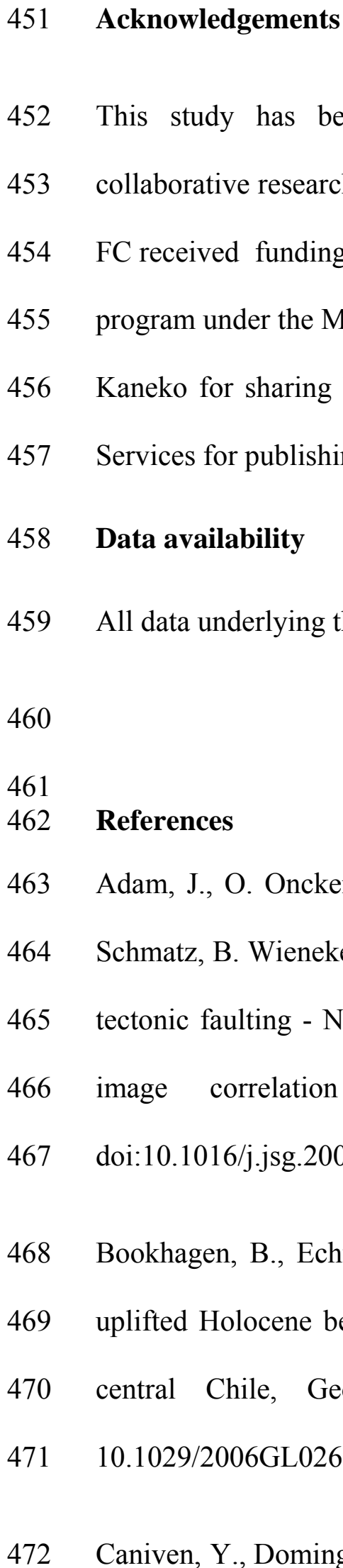

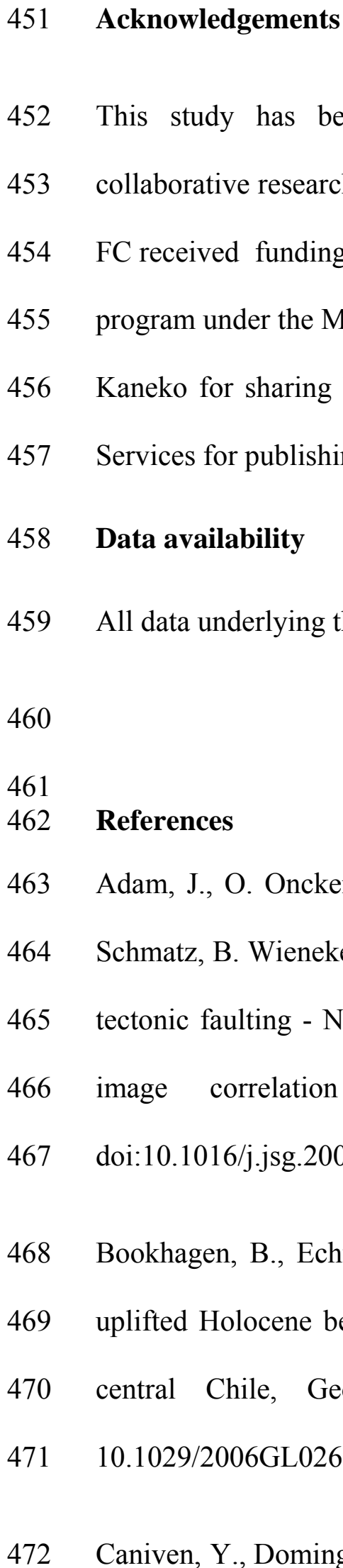

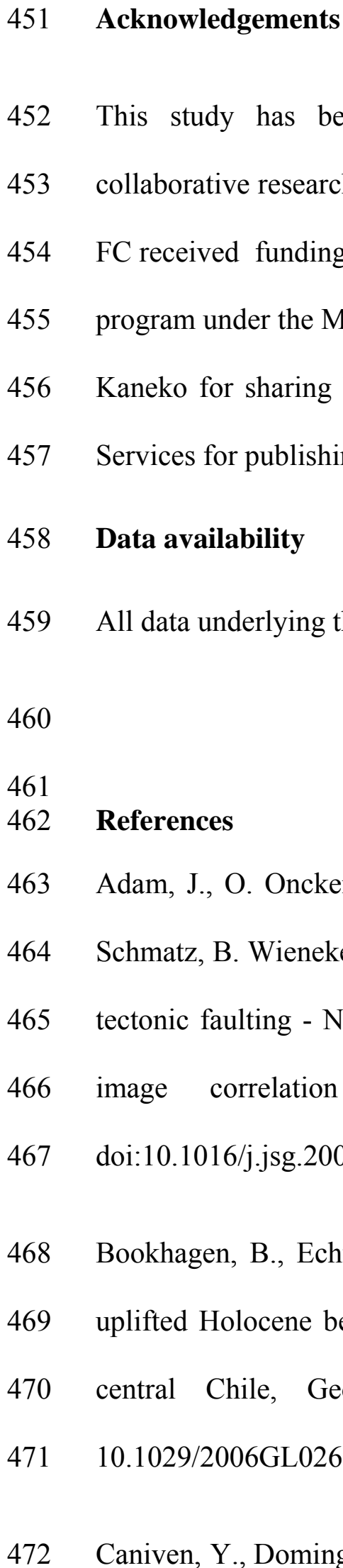

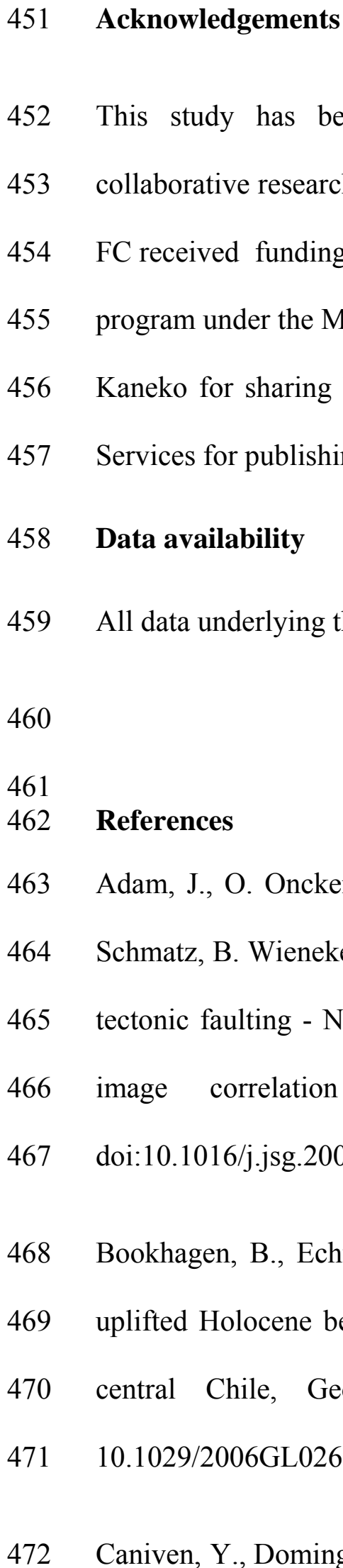

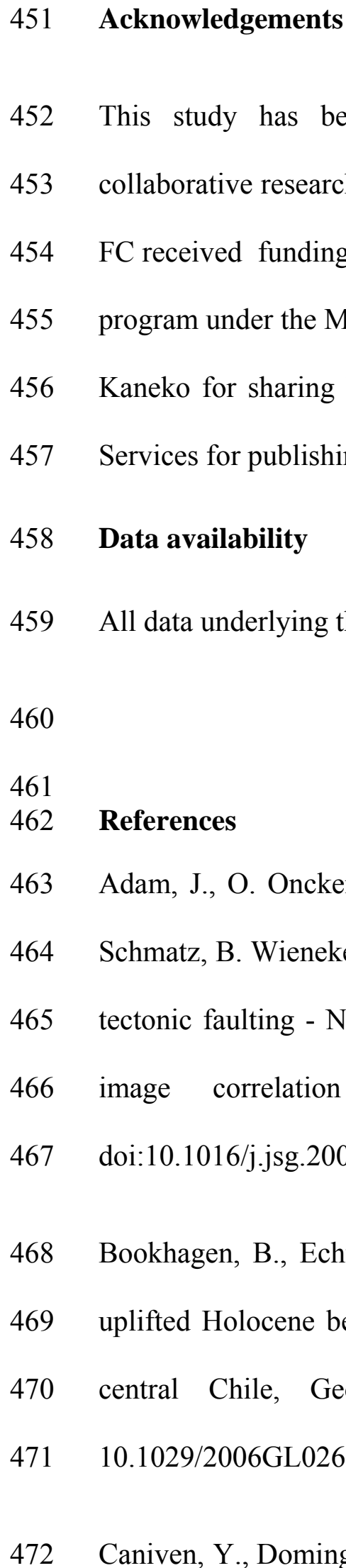

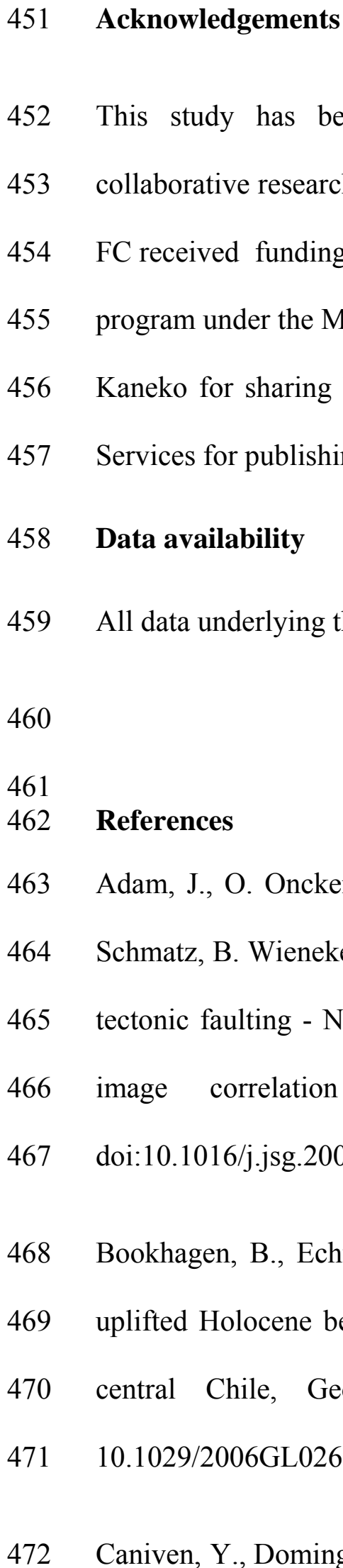

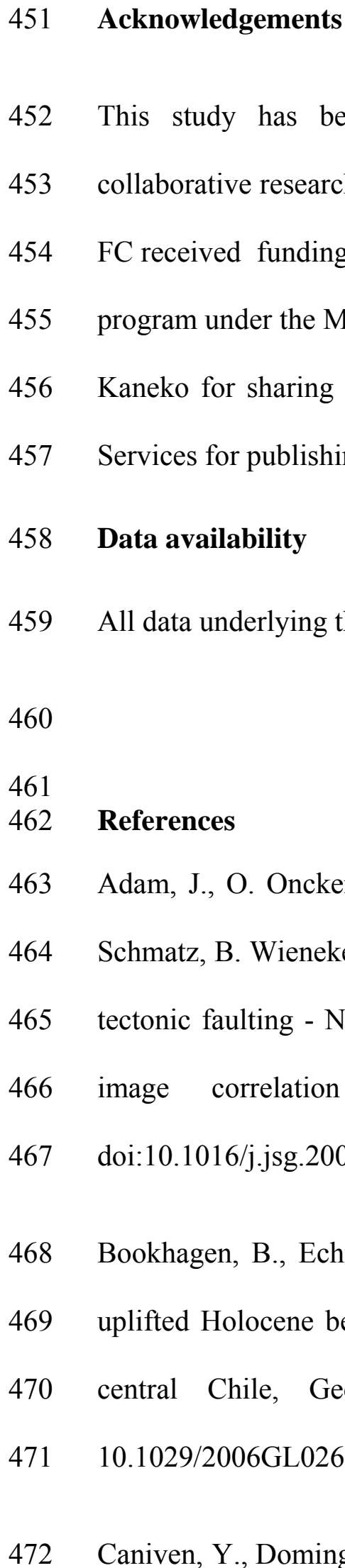

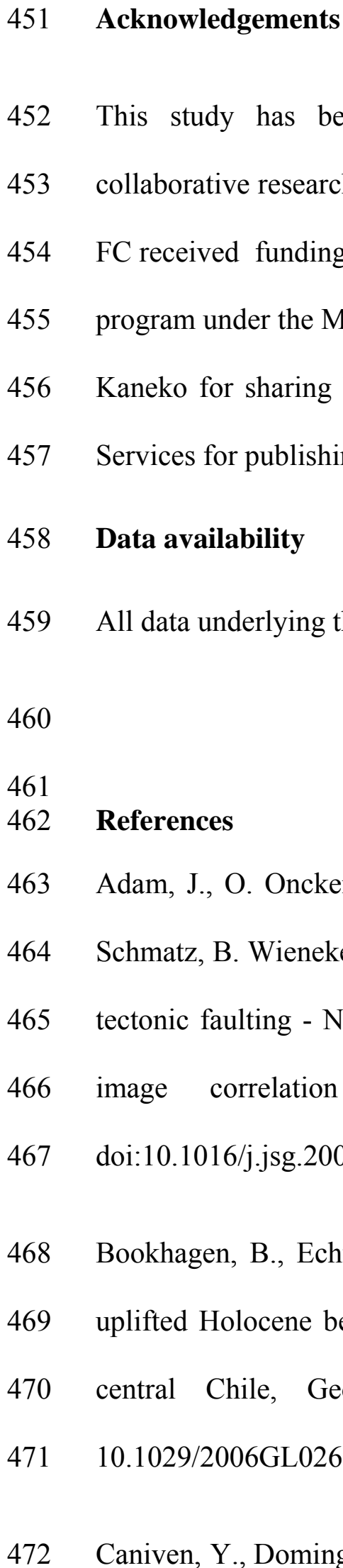

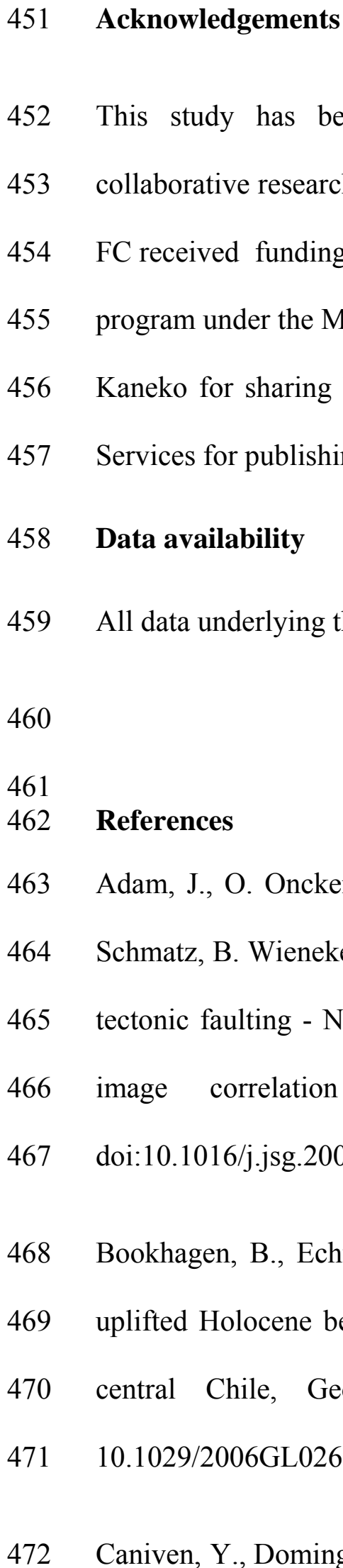

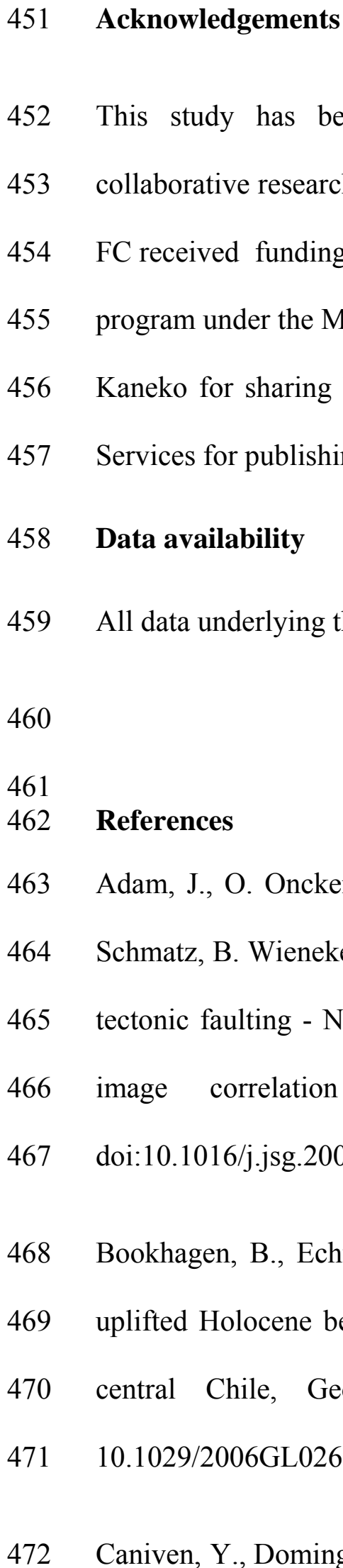

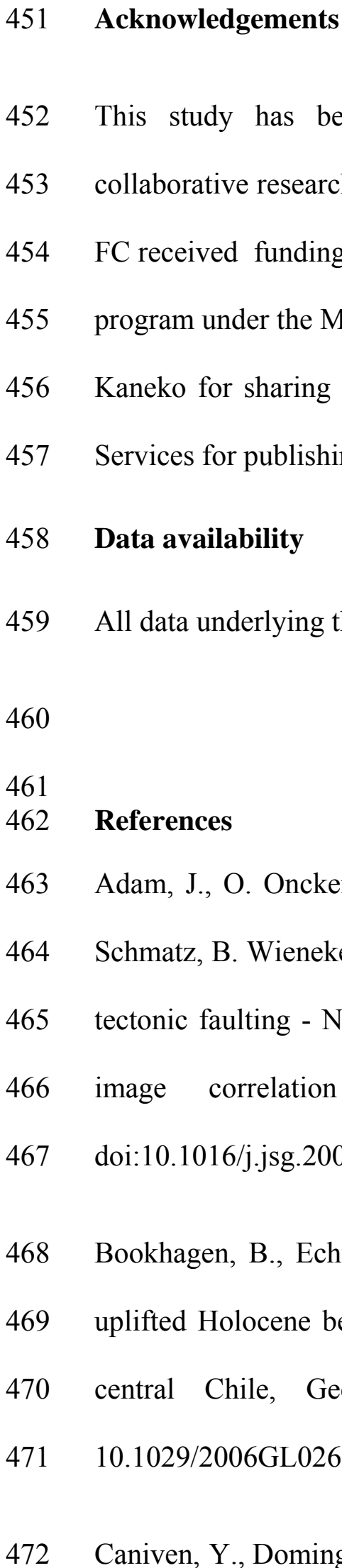

473

474

Caniven, Y., Dominguez, S., Soliva, Roger., Cattin, R., Peyret, M.,Marchandon, M. Romano, C, Strak, V. (2015). A new multilayered visco-elasto-plastic experimental model to study strike-slip fault seismic cycle. Tectonics, 34, 232-264, doi:1002/2014TC003701. 
475 Caniven,Y. Stéphane Dominguez, Roger Soliva, Michel Peyret, Rodolphe Cattin, Frantz 476 Maerten, (2017), Relationships between along-fault heterogeneous normal stress and fault slip 477 patterns during the seismic cycle: Insights from a strike-slip fault laboratory model, In Earth 478 and Planetary Science Letters, Volume 480, Pages 147-157, ISSN 0012-821X, 479 https://doi.org/10.1016/j.eps1.2017.10.009.

480 Comte, D. \& Pardo, M. Reappraisal of great historical earthquakes in the northern 481 Chile and southern Peru seismic gaps. Nat. Hazards 4, 23-44 (1991).

482 Corbi, F., F. Funiciello, M. Moroni, Y. van Dinther, P. M. Mai, L. A. Dalguer, and C. 483 Faccenna (2013), The seismic cycle at subduction thrusts: 1. Insights from laboratory 484 models, J. Geophys. Res. Solid Earth, 118, 1483-1501, doi:10.1029/2012JB009481.

485 Corbi, F., F. Funiciello, S. Brizzi, S. Lallemand, and M. C. G. L. Rosenau (2017): Control of 486 asperities size and spacing on seismic behavior of subduction megathrusts, Geopys. Res. 487 Letters, 44,

488 Franzke, C., O'Kane, T., Monselesan, D., Risbey, J. and Horenko,I. (2015), Systematic 489 Attribution of Secular Southern Hemispheric Circulation Trends with Observational Forcing 490 Data, Nonlin. Proc. Geophys., 22: 513-525.

491 Goldfinger, C., et al. (2003), Holocene earthquake records from the Casca-dia subduction 492 zone and northern San Andreas fault based on precisedating of offshore turbidites, Annu. Rev. 493 Earth Planet. Sci., 31, 555 - 577,doi:10.1146/annurev.earth.31.100901.141246

494 Hayes, G.P. (2017), The finite kinematic rupture properties of great-sized earthquakes since 495 1990, Earth and Planetary Science Letters, Volume 468, Pages 94-100, 496 https://doi.org/10.1016/j.eps1.2017.04.003. 
497 Horenko, I. (2009), On robust estimation of low-frequency variability trends in discrete

498 Markovian sequences of atmospheric circulation patterns. J. of Atmos. Sci., 66(11):19414991954.

500 Horenko, I. (2010), Finite element approach to clustering of multidimensional time series.

501 SIAM J. of Sci. Comp., 32(1):62-83.

502 Hubbert, M. K. (1937), Theory of scale models as applied to the study of geological 503 structures, Geological Society America Bulletin, 48, 459-1520.

504 Kaiser, O. Igdalov, D. and Horenko, I. (2015), "Statistical regression analysis of threshold 505 excesses with systematically missing covariates." SIAM Multiscale Modeling \& Simulation 506 (SIAM MMS), 13(2):594-613.

507 O’Kane, T. , Risbey, J. ,Monselesan, D., Horenko, I. and Franzke, C. (2016), “On the 508 dynamics of persistent states and their secular trends in the waveguides of the southern 509 hemisphere troposphere.” Climate Dynamics, 46(11-12):3567-3597.

510 Risbey, J., O'Kane, T., Monselesan, D, Franzke, C. and Horenko, I. (2015), “Metastability of 511 Northern Hemisphere teleconnection modes.” J. Atmos. Sci., 72:35-54.

512 Kaneko, Y., J. P. Avouac, and N. Lapusta (2010), Towards inferring earthquake patterns from 513 geodetic observations of interseismic coupling, Nature Geoscience, 3(5), 363-U324, 514 doi:10.1038/ngeo843.

515 King, G. C. P., R. S. Stein, and J. Lin (1994), Static stress changes and the triggering of 516 earthquakes, Bulletin of the Seismological Society of America, 84(3), 935-953. 
517 Kuehn, N. M., S. Hainzl, and F. Scherbaum (2008), Non-Poissonian earthquake occurrence in 518 coupled stress release models and its effect on seismic hazard, Geophys. J. Int., 174, 649-658, 519 doi:10.1111/j.1365-246X.2008.03835.X.

Lin, J., Stein, R.S. (2004), Stress triggering in thrust and subduction earthquakes and stress

560 interaction between the southern San Andreas and nearby thrust and strike-slip faults, Journal 561 of Geophysical Research, 109, doi:10.1029/2003JB002607.

562 Metzner, P Putzig, L. and Horenko, I. (2012) “Analysis of persistent non-stationary time 563 series and applications.” Comm. in Appl. Math. and Comp. Sci. (CAMCoS), 7(2):175-229.

564 Moreno, M. S., J. Bolte, J. Klotz, and D. Melnick (2009), Impact of megathrust geometry on 565 inversion of coseismic slip from geodetic data: Application to the 1960 Chile 566 earthquake, Geophys. Res. Lett., 36, L16310, doi: 10.1029/2009GL039276.

567 Moreno, M., Rosenau, M., Oncken, O. (2010), 2010 Maule earthquake slip correlates with 568 pre-seismic locking of Andean subduction zone, Nature, 467 (7312), pp. 198-202 DOI: $569 \quad 10.1038 /$ nature09349

570 Moreno, M., D. Melnick, M. Rosenau, J. Bolte, J. Klotz, H. Echtler, J. Baez, K. Bataille, J. 571 Chen, M. Bevis, H. Hase, O. Oncken (2011), Heterogeneous plate locking in the South572 Central Chile subduction zone: Building up the next great earthquake, Earth and Planetary 573 Science Letters, Volume 305, Issues 3-4, Pages 413-424, 574 https://doi.org/10.1016/j.eps1.2011.03.025.

575 Okada, Y. (1985), Surface deformation due to shear and tensile faults in a half-space, Bulletin 576 of the Seismological Society of America, 75(4), 1135-1154.

577 Okada, Y. (1992), Internal deformation due to shear and tensile faults in a half-space, Bulletin 578 of the Seismological Society of America, 82(2), 1018-1040. 
579 Rosenau, M., F. Corbi, and S. Dominguez (2017), Analogue earthquakes and seismic cycles:

580 Experimental modelling across timescales, Solid Earth, 8, 3, 1-65, doi.: 10.5194/se-8-597$581 \quad 2017$

582 Rosenau, M., J. Lohrmann, and O. Oncken (2009), Shocks in a box: An analogue model of 583 subduction earthquake cycles with application to seismotectonic forearc evolution, Journal of 584 Geophysical Research-Solid Earth, 114, 20, doi: 10.1029/2008jb005665.

585 Rosenau, M., R. Nerlich, S. Brune, and O. Oncken (2010), Experimental insights into the 586 scaling and variability of local tsunamis triggered by giant subduction megathrust 587 earthquakes, Journal of Geophysical Research-Solid Earth, 115, doi:10.1029/2009jb007100.

588 Rosenau, M., and O. Oncken (2009), Fore-arc deformation controls frequency-size 589 distribution of megathrust earthquakes in subduction zones, Journal of Geophysical Research$590 \quad$ Solid Earth, 114, 12, doi:10.1029/2009jb006359.

591 Rosenau et al. (2018): Supplement to "Synchronization of great subduction megathrust 592 earthquakes: Insights from scale model analysis", GFZ Data Services. 593 http://doi.org/10.5880/GFZ.4.1.2018.---

594 Ruff, L. J. (1996), Large Earthquakes in Subduction Zones: Segment Interaction and 595 Recurrence Times, in Subduction Top to Bottom, edited, pp. 91-104, American Geophysical 596 Union.

597 Scholz, C. H. (1998), Earthquakes and friction laws, Nature, 391, 37-42.

598 Shearer, P.M., Stark, P.B. (2012), Global risk of big earthquakes has not recently increased, 599 Proceedings of the National Academy of Sciences of the United States of America, 109 (3), 600 pp. 717-721. DOI: $10.1073 /$ pnas. 1118525109 
601 Shreve, R. L., and Cloos, M. (1986), Dynamics of sediment subduction, melange formation, 602 and prism accretion, Journal of Geophysical Research, 91, 10229-10245.

603 Sykes, L. R., and W. Menke (2006), Repeat times of large earthquakes: Implications for 604 earthquake mechanics and long-term prediction, Bull.Seismol. Soc. Am., 96(5), 1569 - 1596, 605 doi:10.1785/0120050083.

606 Toda, S., Stein, R. S. (2002), Response of the San Andreas fault to the 1983 Coalinga-Nuñez 607 Earthquakes: An application of interaction-based probabilities for Parkfield, Journal of 608 Geophysical Research, 107, doi:0.1029/2001JB000172.

609 Vercauteren, N., Klein, R. (2015). A clustering method to characterize intermittent bursts of 610 turbulence and interaction with submeso motions in the stable boundary layer. J. Atmos. Sci., $61172,1504-1517$

612 

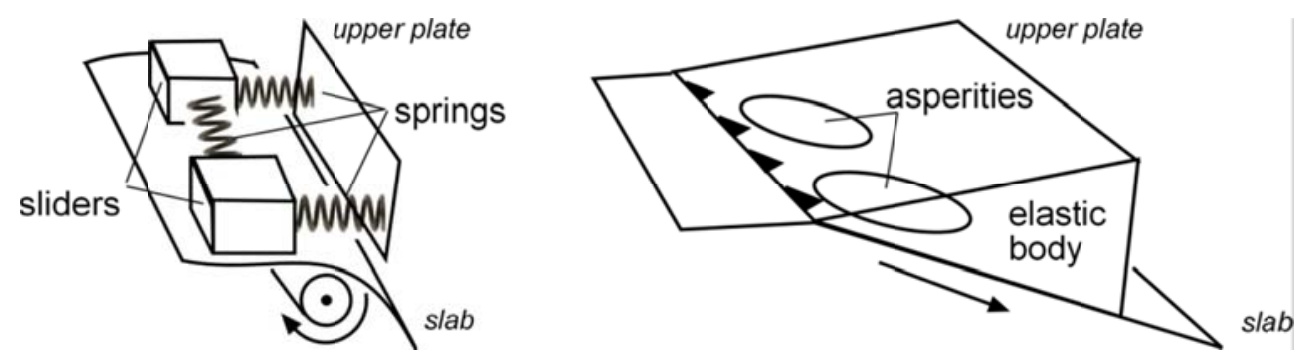

615 Figure 1: The concept of stress coupling and synchronization in subduction zones by means of coupled spring

616 sliders as depicted by Ruff (1996) and the modern transformation of the idea by means of asperities coupled by 617 elastic stress transfer in an elastic medium (upper plate wedge).

618 


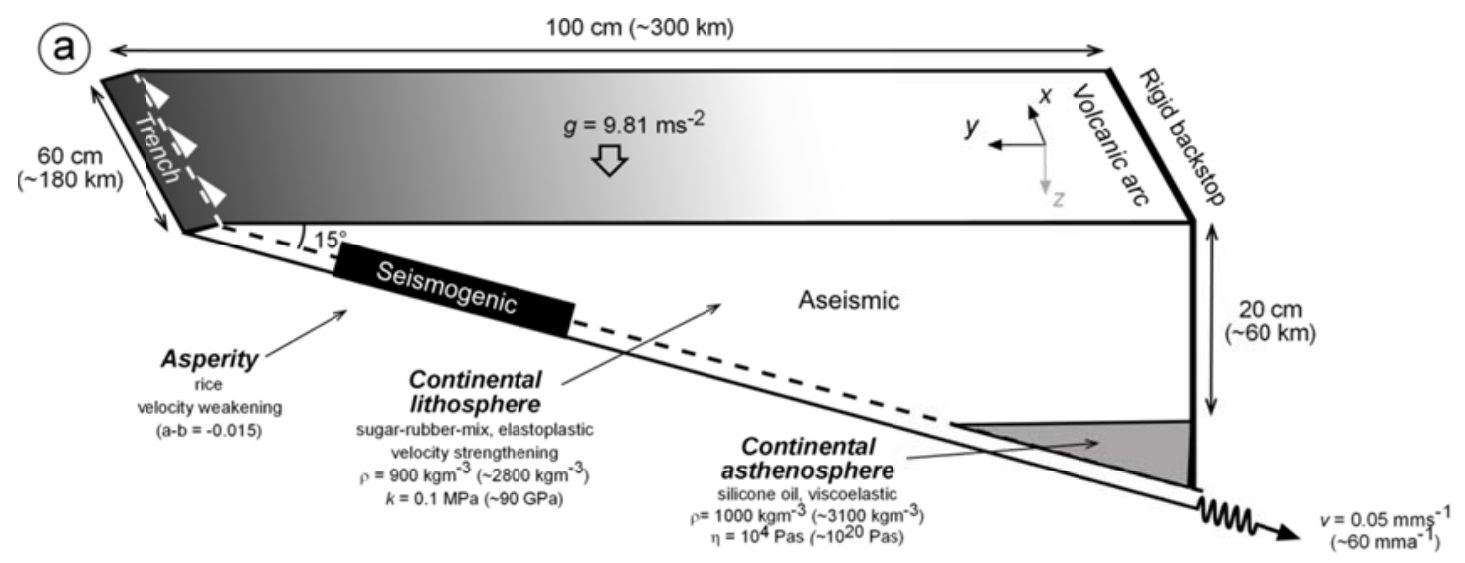

(b)

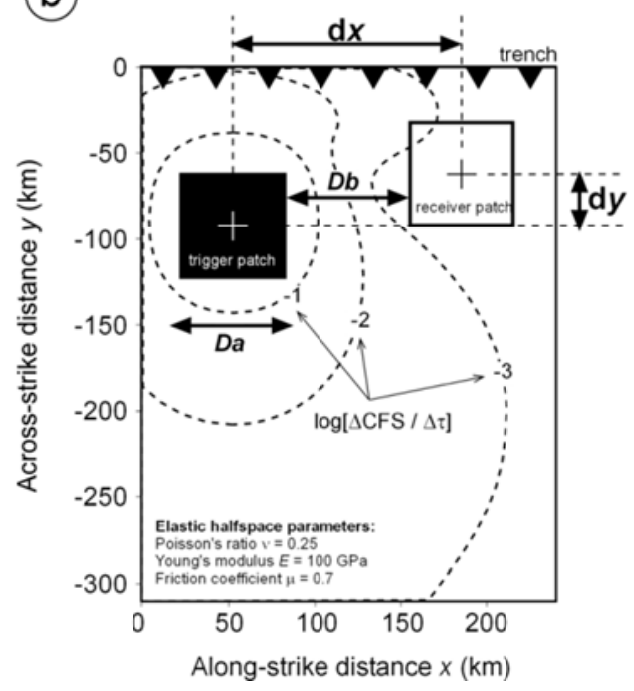

(C)

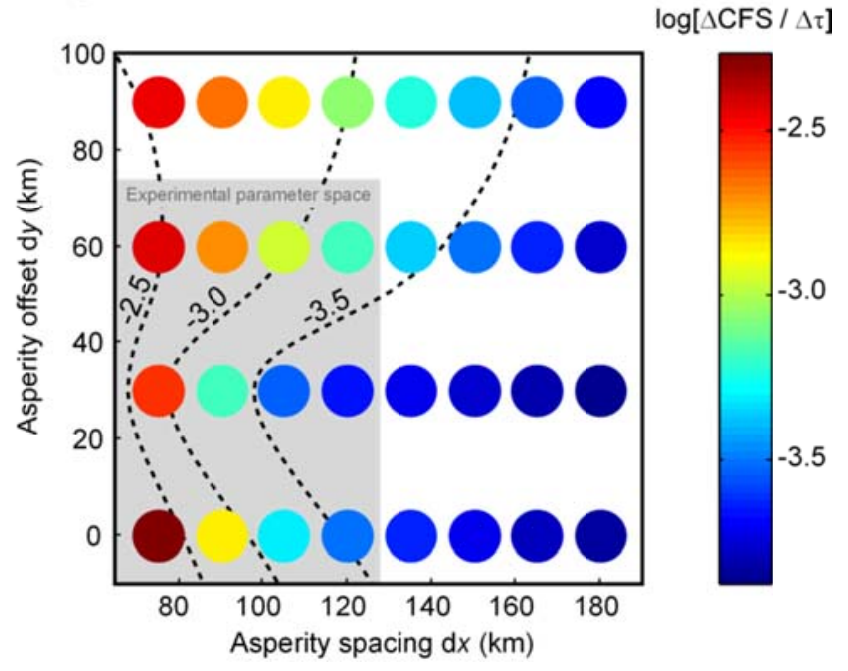

Figure 2: Seismotectonic scale model setup: (a) 3D view of analogue model setup (cross-section corresponds to

$621 x=50 \mathrm{~km}$ in (b)); (b) Map-view (surface projection) of megathrust setup with calculated Coulomb stress

622 changes dCFS (normalized to stress drop dTau on trigger asperity) indicated (note the logarithmic fall-off with

623 distance from the trigger asperity). Da and Db refer to the parameters used by Corbi et al. (2017). (c)

624 Parameter space: Asperity spacing (dx) and offset (dy) and corresponding stress coupling log(dCFS/dTau) in

625 color and isolines. Grey shaded area corresponds to the subspace realized experimentally. Size of the asperities

626 has not been changed in this study. 
(a)

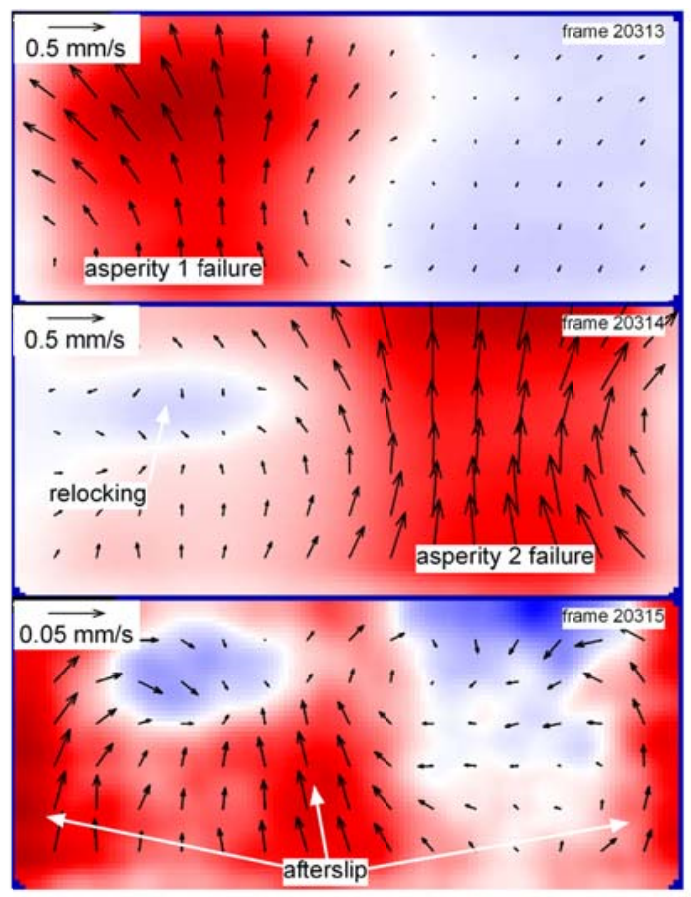

(b)

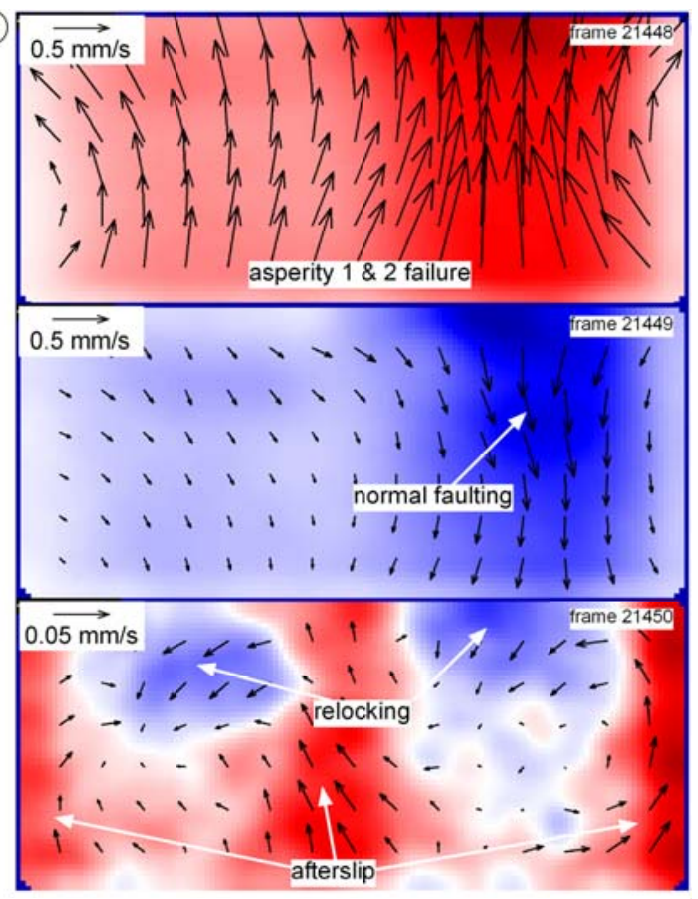

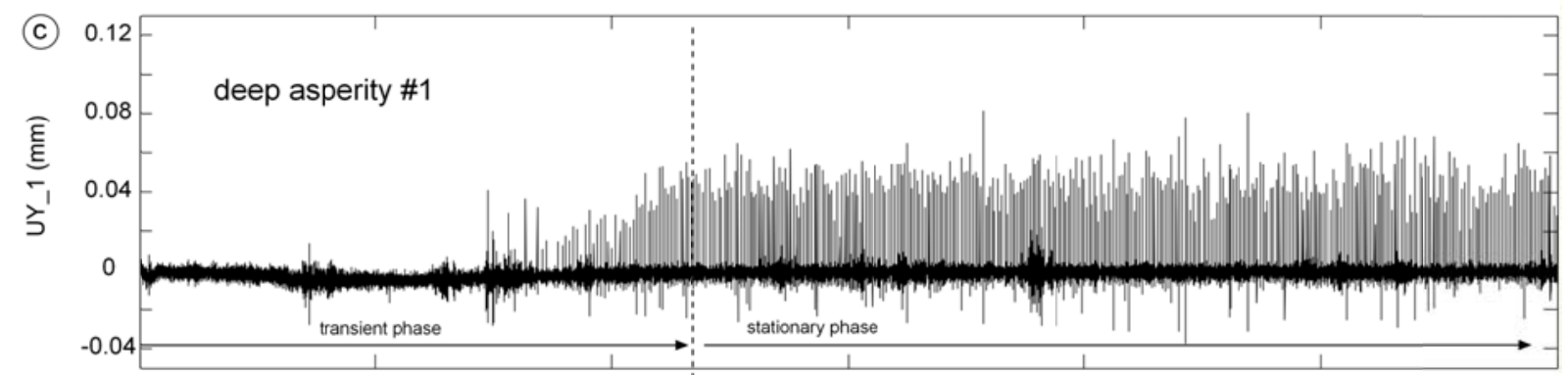

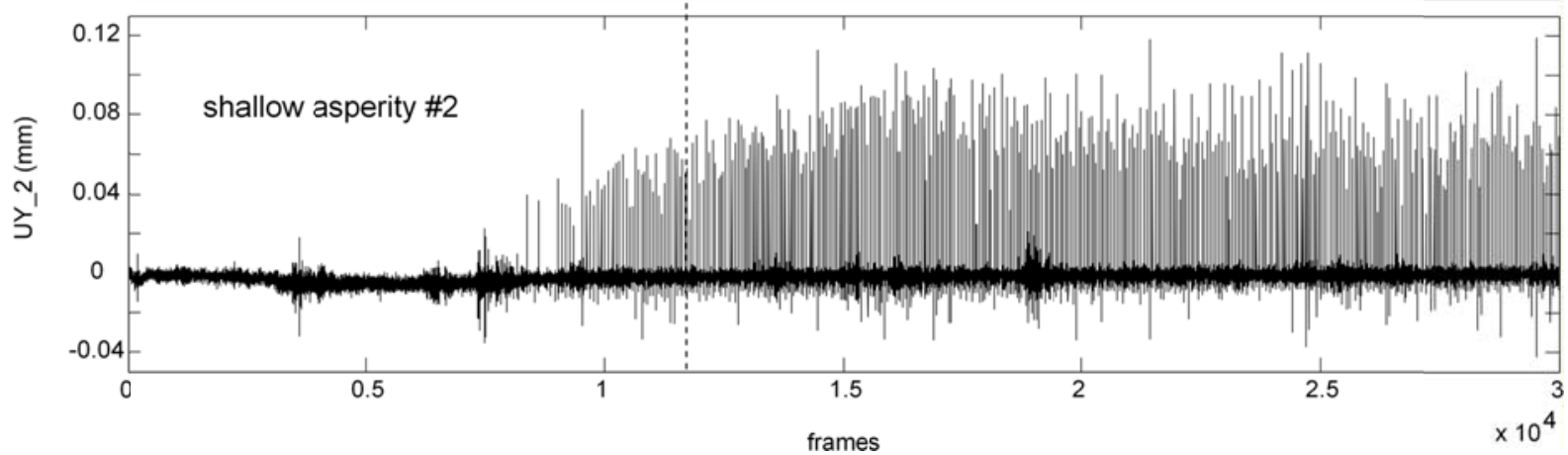

Figure 3: Example of surface deformation pattern on top of the asperities (trench is north): (a) sequence of a

630 cluster of two solo events followed by afterslip and relocking (each velocity field corresponds to 0.1 second experimental time). (b) sequence of a double event followed by a normal event, relocking and afterslip. Note the different vector scale for coseismic (upper, middle panel) and postseismic phases. Colors are scaled to the maximum velocity in each panel $(r e d=$ surface displacement towards trench, white $=0$, blue $=$ away from the trench). (c) time-series of surface deformation towards the trench (UY) averaged over the surface projected area of asperity 1 and asperity 2 used for further analysis. Note the asymmetry in displacements above shallow and deep asperity which is related to the free-surface effect. 
(a)

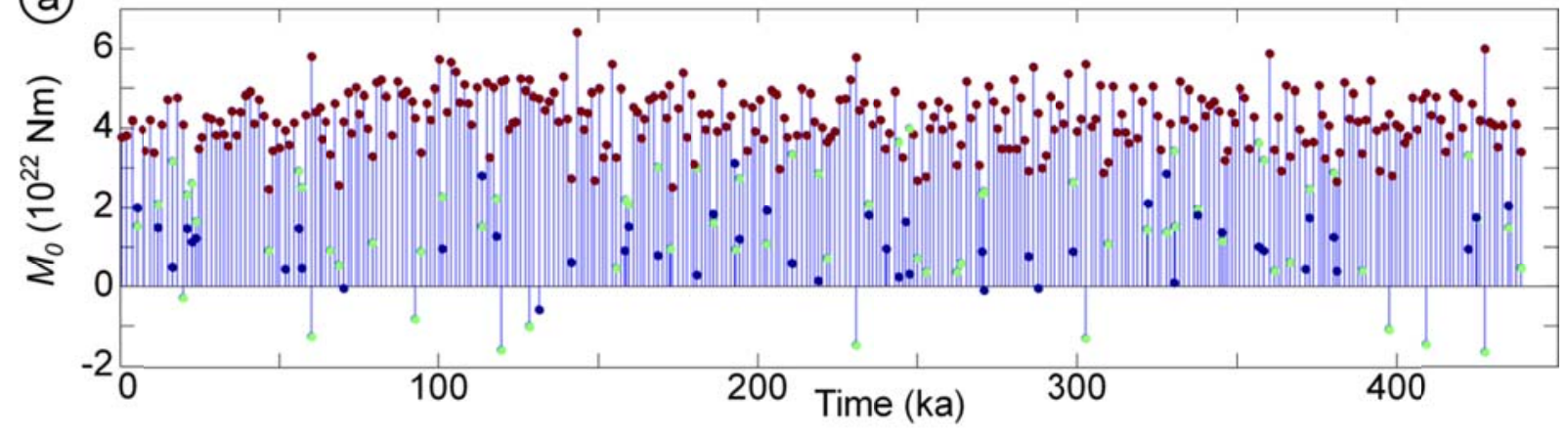

(b)

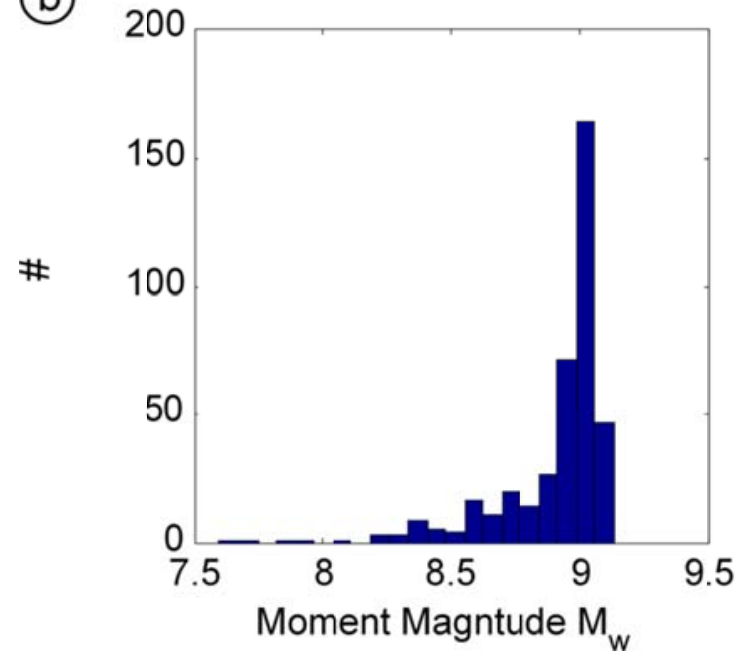

(C)

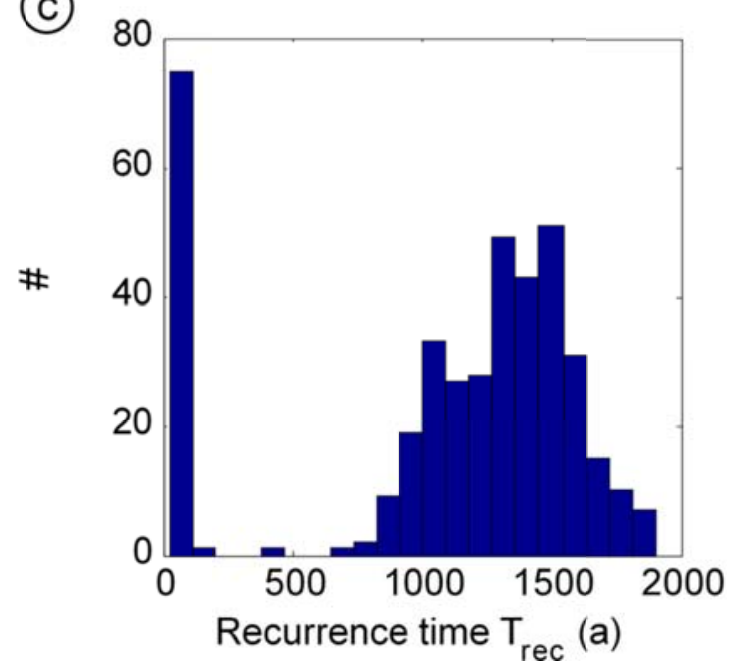

639 Figure 4: Example of an earthquake sequence simulated using seismotectonic scale modelling and derived by 640 the numerical FEM-BV approach (all parameters scaled to nature): (a) event catalogue: (b) pdf of moment 641 magnitude $M w$, (c) pdf of recurrence time Trec. See appendix figure A3 for pdfs of all experiments. 
(a)

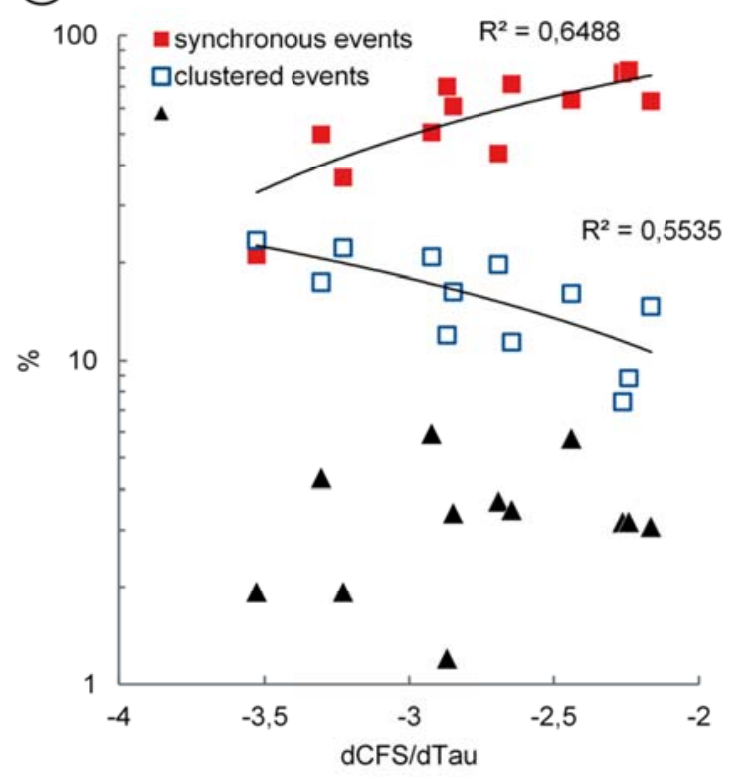

(b)

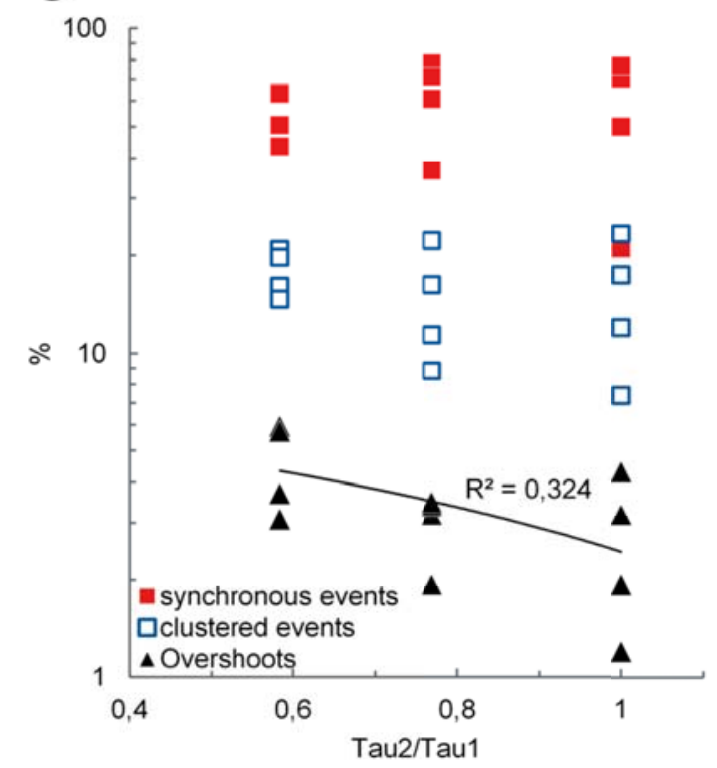

643

$644 \quad$ Figure 5: Percentage of different types of events versus stress coupling (a) and strength contrast (b).

645 

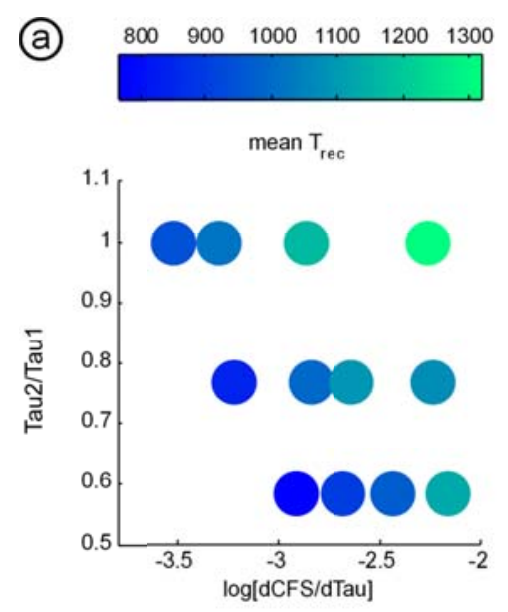

(C)

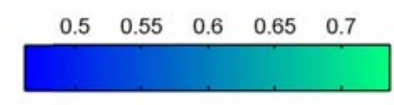

$C V T_{\text {rec }}$

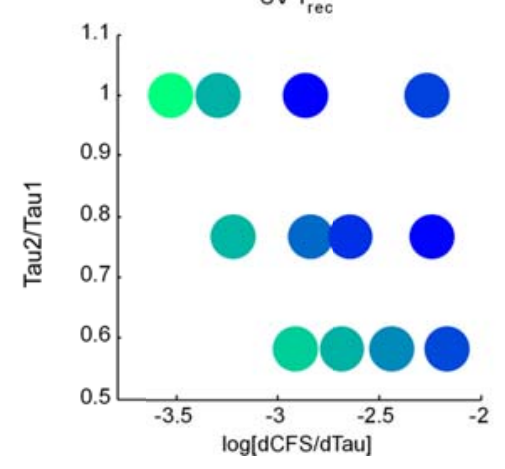

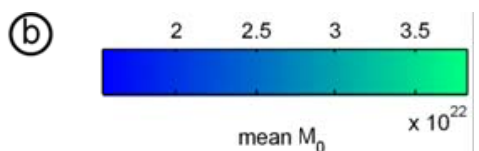
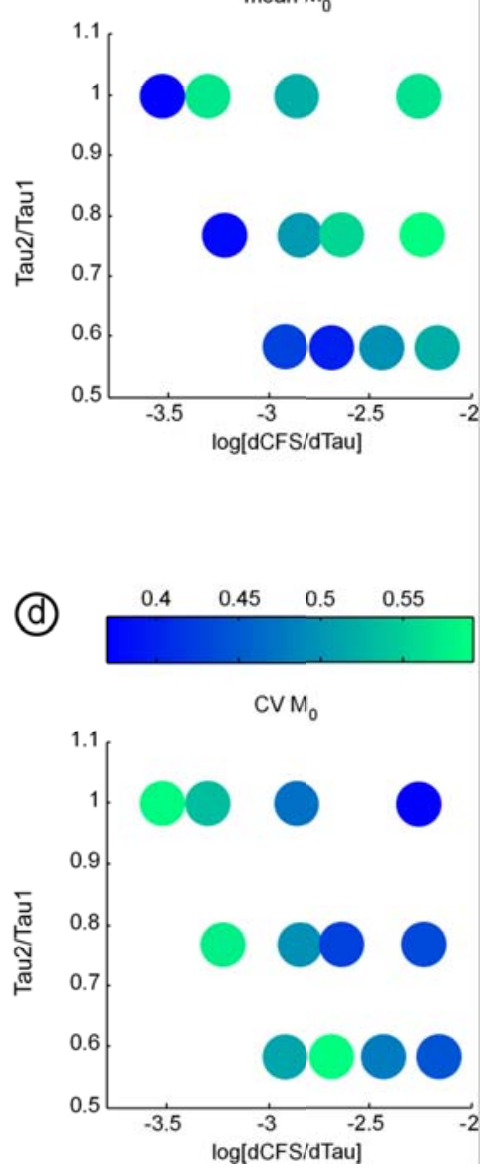

646

647 Figure 6: Correlation between of recurrence time $(a, c)$ and seismic moment $(b, d)$ with stress coupling 648 (dCFS/dTau) and strength contrast (Tau2/Tau1). See table A1 for regression analysis results. 

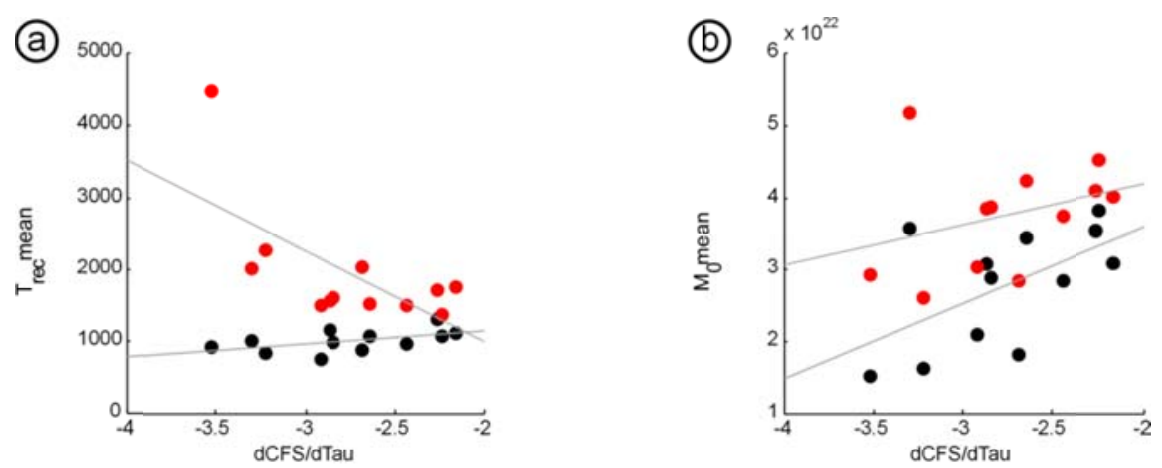

- single \& double events double events

650

(C)

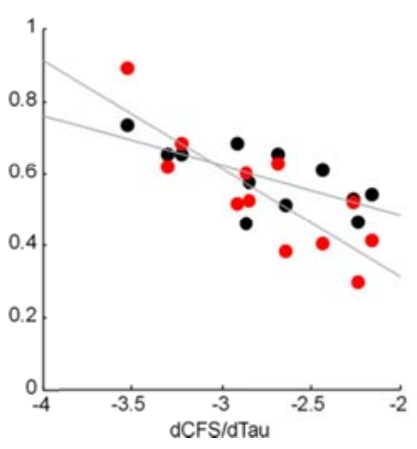

(d)

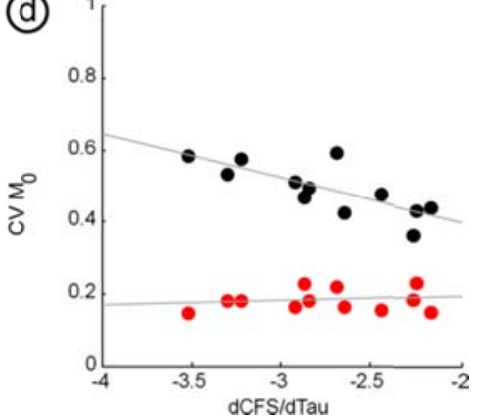

$651 \quad$ Figure 7: Correlation between recurrence time (a) and its CV (c) and seismic moment (b) its CV (d) with stress 652 coupling (dCFS/dTau) for all events (black dots) and double events (red dots). 


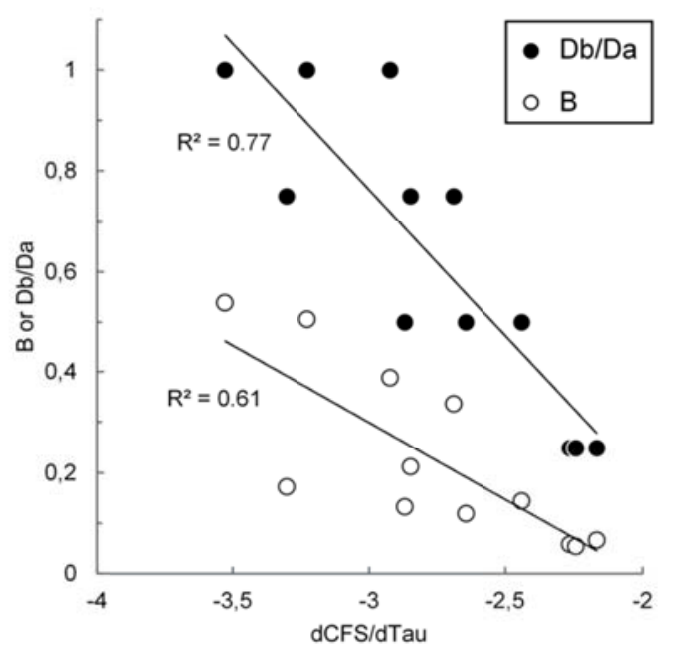

655 Figure 8: Correlation of stress coupling with B and Db/Da parameters.

656 

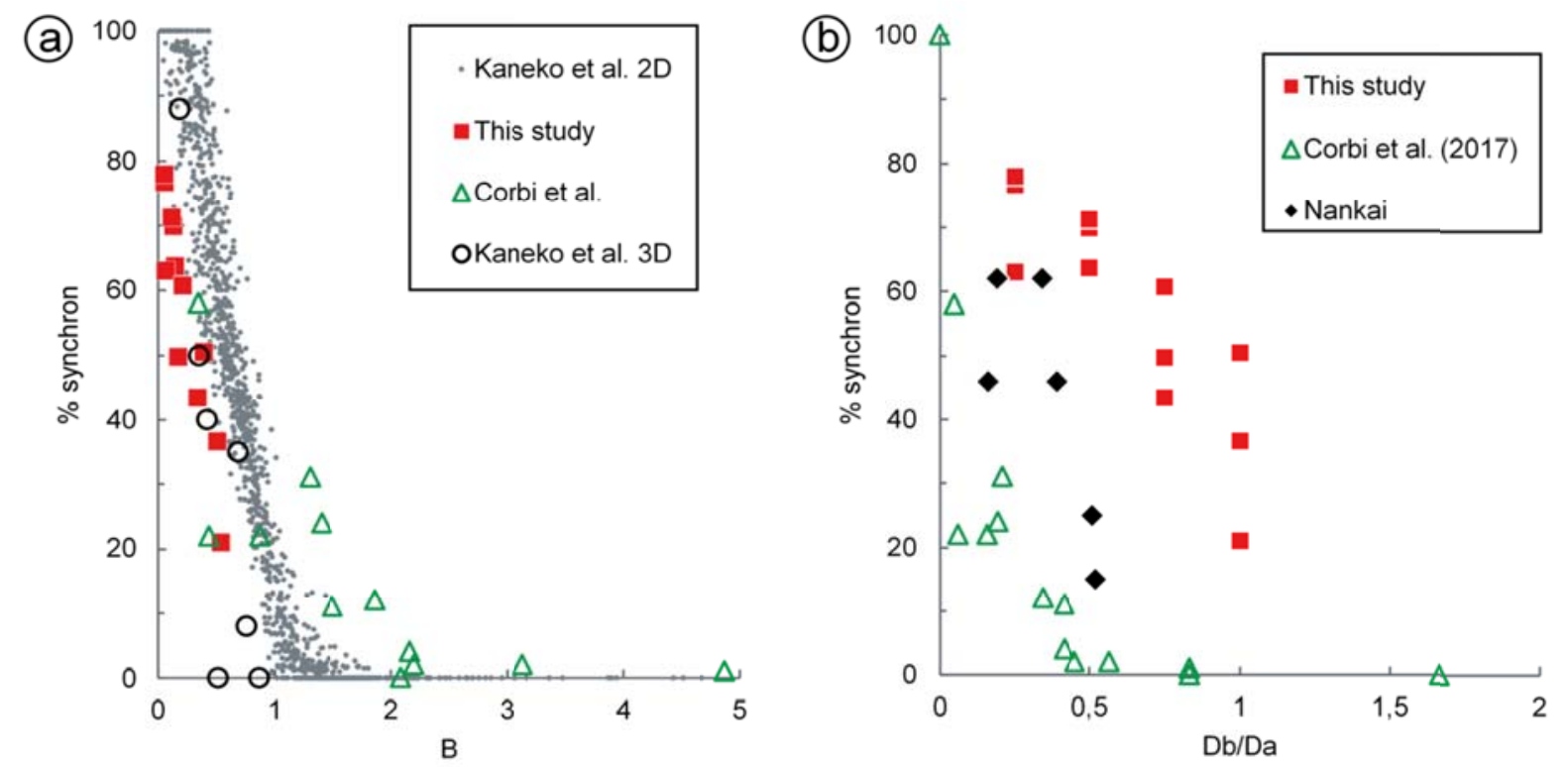

658 Figure 9: Probability of synchronous events as a function of $B$ (a) and Db/Da (b). Note collapse of 659 experimental, natural and numerical simulation data in (a). Parallel offset trends in (b) are interpreted as due to 660 differences in frictional properties between the experiments and nature. 


\section{Parameters:}

\begin{tabular}{llll} 
Quantity & $\begin{array}{l}\text { Symb } \\
\text { ol }\end{array}$ & $\begin{array}{l}\text { Dimensio } \\
n\end{array}$ & Unit \\
& & M,L,T $\}$ & \\
\hline
\end{tabular}

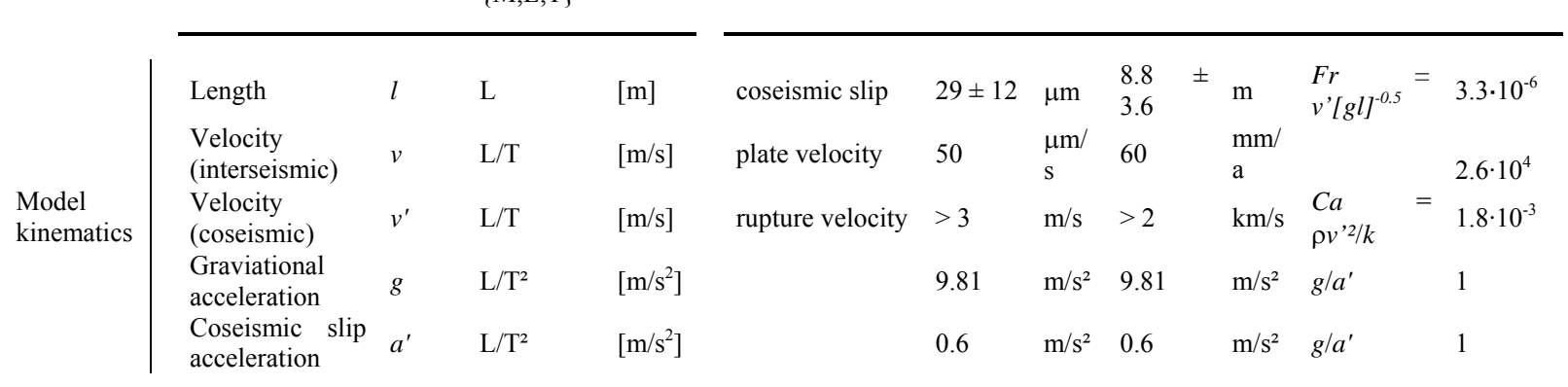

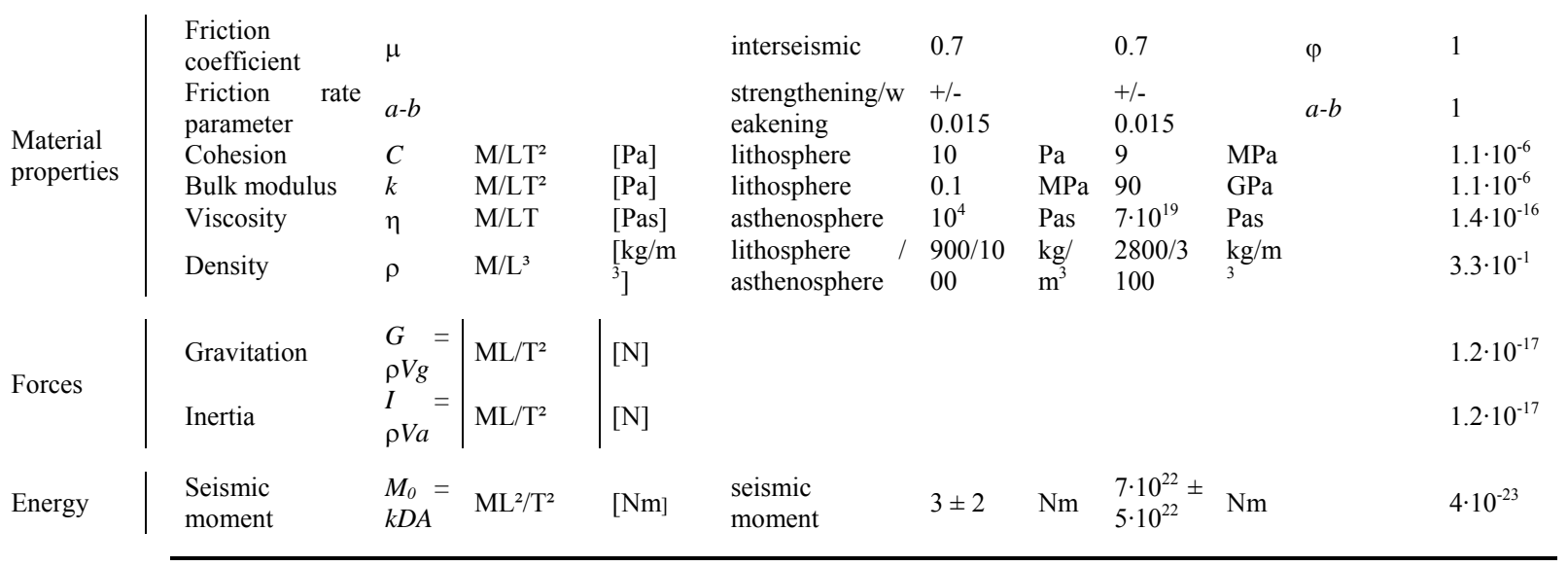

Similarity:

Quantitiy Model Nature Dimensionles Scaling

Nature number factor

663 Table 1: Analogue model parameters, scaling relations and material properties 
665

666

667

668

669

670

671

672

\section{APPENDIX}

\begin{tabular}{|l|l|l|}
\hline Parameter $\boldsymbol{X}$ & Parameter $\boldsymbol{Y}$ & $\boldsymbol{R}^{\mathbf{2}}$ \\
\hline dCFS/DTau & $T_{\text {rec }}$ & 0.285 \\
\hline dCFS/DTau & $M_{0}$ & 0.333 \\
\hline dCFS/DTau & CV $T_{\text {rec }}$ & 0.475 \\
\hline dCFS/DTau & CV $M_{0}$ & 0.588 \\
\hline Tau1/Tau2 & $T_{\text {rec }}$ & 0.245 \\
\hline Tau1/Tau2 & $M_{0}$ & 0.055 \\
\hline Tau1/Tau2 & CV $T_{\text {rec }}$ & 0.012 \\
\hline Tau1/Tau2 & CV $M_{0}$ & 0.010 \\
\hline
\end{tabular}

Table A1: Results from linear regression analysis (green = statistically significant; red = insignificant).See

Figure 6 for visulalization of trends. 
Fig. A1

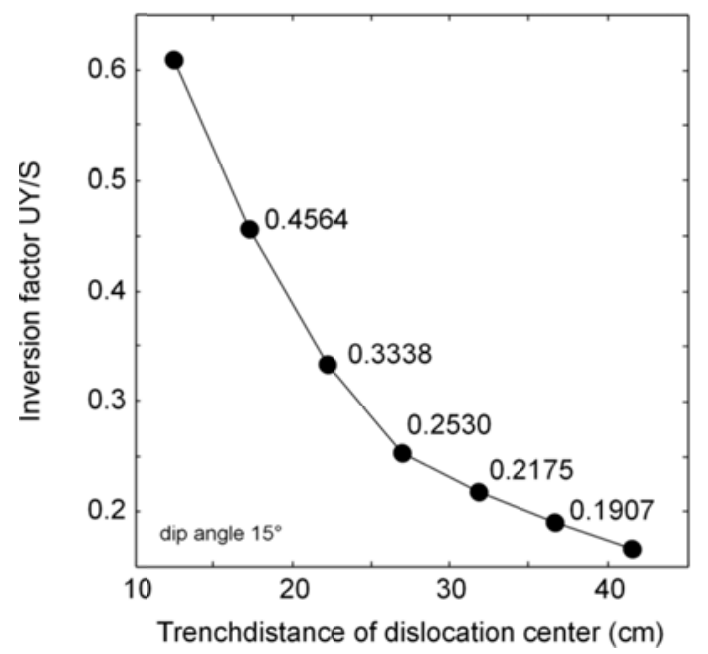

674 Figure A1: Relation between horizontal surface displacement and slip on dislocation as a function of trench 675 distance (depth). 
Fig.A2

676

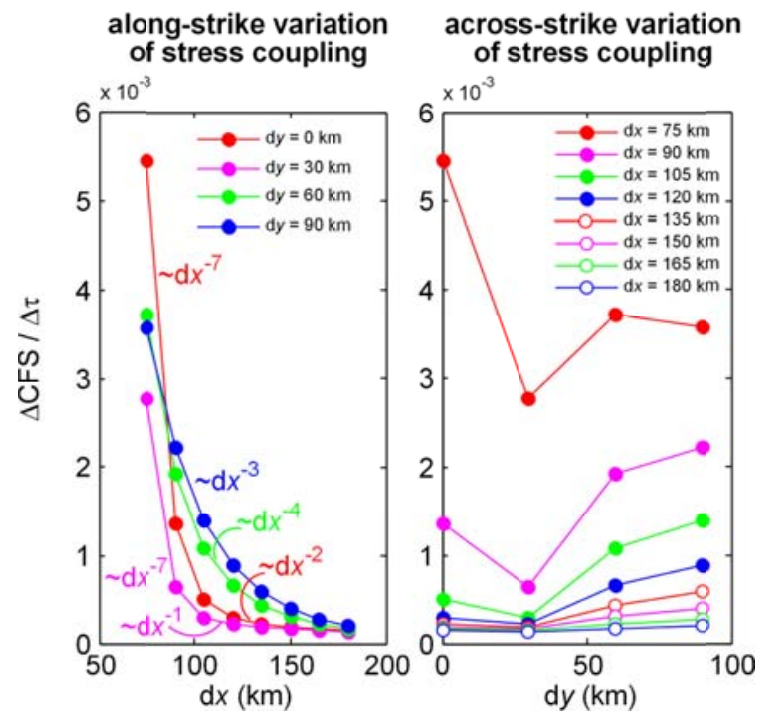

677 Figure A2: Spatial variation of coulomb stress transfer along strike and across strike of the subduction zone as

678 predicted by elastic dislocation modelling. Definition of $d x$ and dy see main text. 
Fig. A3
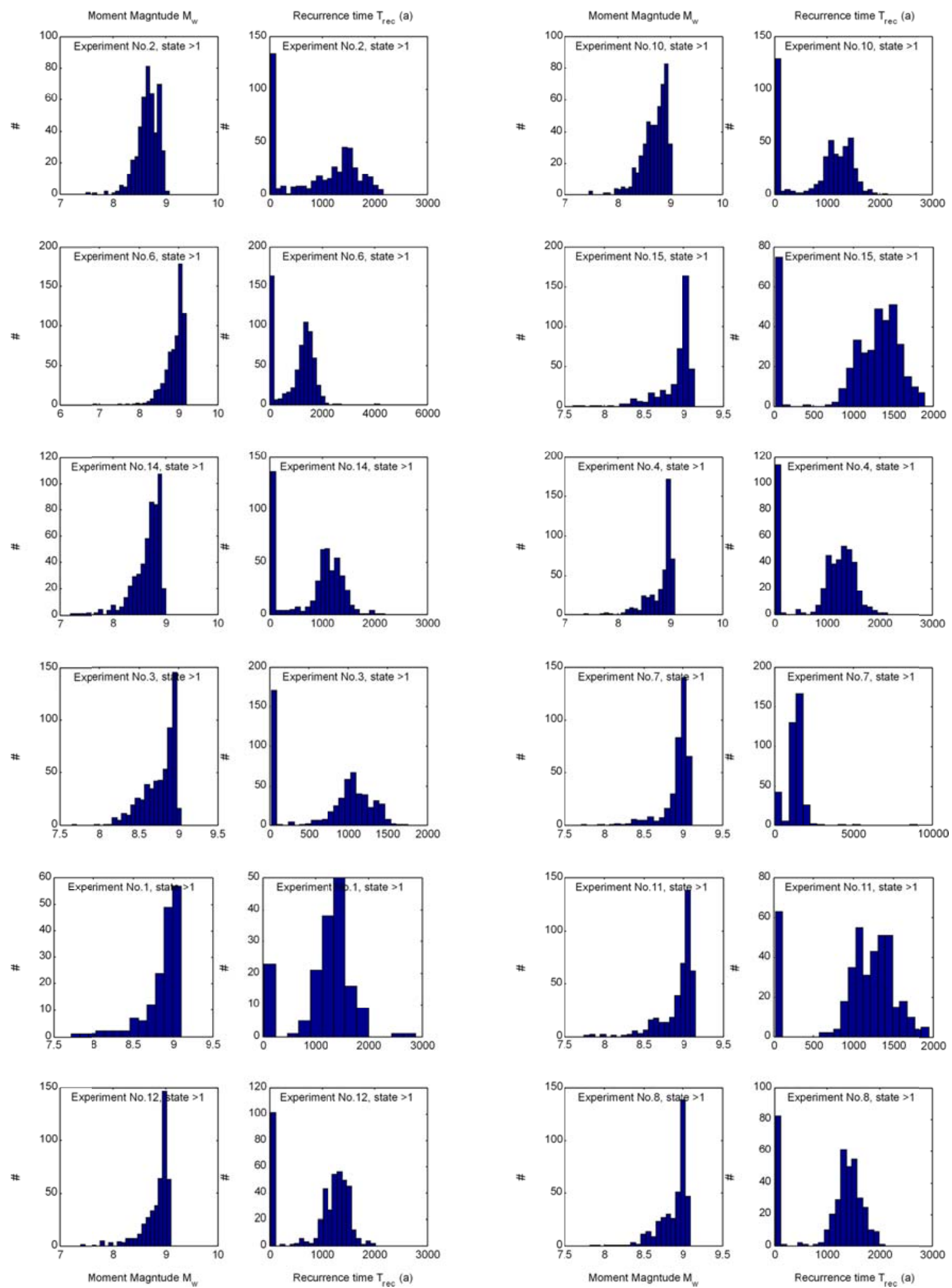

680 Figure A3: Probability distribution functions ( $p d f s)$ of $M w$ and Trec for all experiments. The order of the plots is

681 such that in the two rows experiments increase in stress coupling downwards. Second row is continuation of first row. 